\title{
Exact analysis of divergent inventory systems with time-based shipment consolidation and compound poisson demand
}

\section{Citation for published version (APA):}

Stenius, O., Karaarslan, A. G., Marklund, J., \& de Kok, A. G. (2016). Exact analysis of divergent inventory systems with time-based shipment consolidation and compound poisson demand. Operations Research, 64(4), 906-921. https://doi.org/10.1287/opre.2016.1510

DOI:

10.1287/opre.2016.1510

Document status and date:

Published: 31/07/2016

\section{Document Version:}

Accepted manuscript including changes made at the peer-review stage

\section{Please check the document version of this publication:}

- A submitted manuscript is the version of the article upon submission and before peer-review. There can be important differences between the submitted version and the official published version of record. People interested in the research are advised to contact the author for the final version of the publication, or visit the $\mathrm{DOI}$ to the publisher's website.

- The final author version and the galley proof are versions of the publication after peer review.

- The final published version features the final layout of the paper including the volume, issue and page numbers.

Link to publication

\section{General rights}

Copyright and moral rights for the publications made accessible in the public portal are retained by the authors and/or other copyright owners and it is a condition of accessing publications that users recognise and abide by the legal requirements associated with these rights.

- Users may download and print one copy of any publication from the public portal for the purpose of private study or research.

- You may not further distribute the material or use it for any profit-making activity or commercial gain

- You may freely distribute the URL identifying the publication in the public portal.

If the publication is distributed under the terms of Article $25 \mathrm{fa}$ of the Dutch Copyright Act, indicated by the "Taverne" license above, please follow below link for the End User Agreement:

www.tue.nl/taverne

Take down policy

If you believe that this document breaches copyright please contact us at:

openaccess@tue.nl

providing details and we will investigate your claim. 


\section{Exact Analysis of Divergent Inventory Systems with Time-Based Shipment Consolidation and Compound Poisson Demand}

Olof Stenius

Department of Industrial Management and Logistics, Lund University, Box 11822100 Lund, Sweden, e-mail:

Olle.Stenius@iml.lth.se

\section{Ayşe Gönül Karaarslan}

Department of Econometrics, Erasmus University Rotterdam, PO Box 1738 3000DR Rotterdam, The Netherlands, Tel: +31-10-4081342, e-mail: Karaarslan@ese.eur.nl

\section{Johan Marklund}

Department of Industrial Management and Logistics, Lund University, Box 11822100 Lund, Sweden, Tel: +46-46-2228013, e-mail:Johan.Marklund@iml.lth.se

\section{A. G. de Kok}

Department of Industrial Engineering and Innovation Sciences, Eindhoven University of Technology, P.O. Box 513 5600MB Eindhoven, The Netherlands, Tel: +31-40-2473849, e-mail: A.G.d.Kok@tue.nl

Keywords: Inventory, Multi-echelon, Multi-item, Stochastic, Shipment consolidation, Continuous review, Compound Poisson demand 


\begin{abstract}
Sustainable and efficient management of a distribution system requires coordination between transportation planning and inventory control decisions. In this context, we consider a one warehouse multi-retailer inventory system with a time-based shipment consolidation policy at the warehouse. This means that there are fixed costs associated with each shipment, and retailer orders are consolidated and shipped periodically to groups of retailers sharing the same delivery routes. Customer demand is compound Poisson distributed and unsatisfied demand at each stockpoint is backordered and allocated on a First-Come First-Served basis. The system is centralized and inventory levels are reviewed continuously. The warehouse has access to real-time inventory information from the retailers, and uses a $(R, n Q)$ policy to replenish from an outside supplier/manufacturer. We derive the exact probability distributions for the inventory levels at the retailers, and use these to obtain exact expressions for the system's expected shipment, holding and backorder costs, its average inventory levels and fill rates. Based on the analytical properties of the objective function, we construct an optimization procedure by deriving bounds on the optimal reorder levels and shipment intervals both for single-item and multi-item systems.
\end{abstract}




\section{Introduction}

The technological development of integrated supply chain information systems is making real-time point-of-sale and inventory information more and more accessible, also across multi-tier supply chains. In the process, administrative costs of sharing information and placing of orders are decreasing, thus reducing the economic incentives for aggregating demand information into orders. On the other hand, set up costs, batch processing, and shipment consolidation are important considerations in manufacturing and distribution of physical products. In freight transportation these issues are accentuated by fluctuating fuel prices and increasing emphasis on environmental concerns and sustainability. With respect to the latter, shipment consolidation can reduce the number of shipments (e.g. trucks) and thereby achieve both lower transportation costs and lower (carbon) emissions. However, consolidation typically means longer replenishment lead times and increased inventory costs. Thus, sustainability in terms of economic viability and environmental friendliness requires coordination and balancing of shipment and inventory decisions.

In this paper, we focus on these issues in the context of a divergent two-echelon inventory system with a central warehouse and multiple non-identical retailers facing compound Poisson demand. The system is characterized by a time-based shipment consolidation policy at the warehouse, in conjunction with real-time point of sale data and centralized inventory information. The consolidation policy means that outbound shipments from the central warehouse are dispatched periodically to groups of retailers sharing the same delivery routes (a group may consist of a single retailer). Thus, retailer orders are consolidated for the retailers on the same route over the time between shipments, referred to as the shipment interval. The delivery routes and grouping of retailers are determined exogenously and optimizing their design is beyond the scope of this paper. Inventory replenishments are based on continuous review information, batch ordering at the warehouse, and base stock ordering at the retailers. The contributions of our work include derivations of the probability distributions for all inventory levels in the system, and determination of the associated expected costs and retailer fill rates. We also provide optimality bounds that allow for joint optimization of the shipment intervals and the reorder levels in the system. The results encompass both the case of backorder costs per unit and time unit at the retailers, and the case of fill rate constraints. For the latter, the total costs only consist of the expected shipment and holding costs. The analysis is applicable to single-item as well as multi-item systems, although the focus in this work is placed on the former. The approach for analyzing the system is new, and can be used for analyzing other types of divergent inventory systems.

Initial motivation for our work stems from discussions with a large European company that manufactures and sells sheet metal products to the construction industry. The company produces both 
to order and to stock, where the former typically involves customized solutions for large construction projects. Focusing on the latter, products are distributed from a central warehouse to a large number of retailers and market companies (hereafter jointly referred to as retailers) using connected IT and inventory control systems. This means that there is centralized access to point-of-sale data and inventory information in real time for the network. Currently, this information is not used for centralized control of the system, but there are ongoing discussions about how this may be done. Many of the company's products are large and bulky with relatively low value to volume ratio. Thus, transportation costs are generally high, meaning that shipment consolidation, and efficient use of the transport capacity are important for the company. Consolidated shipments from the warehouse to the retailers are dispatched periodically to groups of retailers. The transportation planning is performed in-house, but the transports are contracted from external service providers. All outbound transports from the warehouse are made by truck, either a dedicated vehicle delivering to a group of retailers on a specified route, or a shared vehicle utilizing the transport provider's network of terminals. Looking downstream, the majority of the company's customers are construction companies of different sizes, which typically place batch orders dedicated to specific construction projects. As these projects vary in sizes so do the batch sizes. Thus, the customer demand is characterized by randomness both in the number of orders that arrive, and in the size of individual orders. Structurally, this corresponds well to a compound Poisson process, which motivates its use in our present work. Upstream, the warehouse replenishes most of its stock by placing orders with the company's own manufacturing plants.

Further motivation for our work is based on contacts with several other companies (including some spare parts service providers) that use, or are in the position to use, different types of VMI (Vendor Managed Inventory) systems in combination with periodic shipment schedules. (For overviews of different types of VMI systems, see, for example, Cheung and Lee 2002 and Marques et al. 2010.) Although the details may vary, these systems are characterized by a supplier/central warehouse with the mandate to control inventories at different customer locations/retailers under specified service agreements. This control is typically facilitated by access to point-of-sale data and inventory information from the retailers. These centralized systems offer the supplier flexibility in planning production, distribution and replenishing activities. However, a recurring question these companies struggle with is how the centralized inventory information may be used for improved control of their multi-echelon inventory systems.

\section{Literature Review}

Our work is closely related to Marklund (2011), which considers a similar system under the more 
restrictive assumption of Poisson demand. Marklund (2011) presents a fast recursive procedure for determining the exact average costs per time unit, and for optimizing the reorder levels at all inventory locations for a given set of shipment intervals. A heuristic for determining near optimal shipment intervals is also presented. Compared to Marklund (2011) our present work distinguishes itself in three major ways. Firstly, it is more general as we provide an exact approach for compound Poisson demand. Secondly, we use a new methodology for analyzing the system, which enables determination of the inventory level distributions and service levels at all locations, not just the expected costs as in Marklund (2011). Thirdly, we provide upper and lower bounds for the optimal shipment intervals and reorder points in the system, thereby enabling joint optimization of these decision variables.

Howard and Marklund (2011) and Howard (2013) build on the work in Marklund (2011) and use simulation to investigate the cost benefits of replacing the First-Come First-Served (FCFS) allocation assumption at the warehouse with state dependent myopic allocation policies under Poisson demand. Howard and Marklund (2011) deal with one retailer group whereas Howard (2013) considers multiple retailer groups and a policy that offers a performance guarantee over FCFS. Both studies conclude that some cost benefits exist for long retailer lead times, particularly, when the allocation decision is postponed to the moment of delivery, but in general the FCFS assumption performs very well. Gürbüz et al. (2007) also consider joint inventory and transportation decisions, but for a system where the warehouse is a stockless cross docking facility that orders for a set of retailers simultaneously. This policy is compared to three well-known policies including one with fixed replenishment intervals.

Our present work is also related to the general multi-echelon literature which does not take shipment consolidation decisions into consideration. Of particular interest is the stream of literature on analysis of continuous review one-warehouse multiple-retailer systems. For overviews, see for example, Axsäter (2003) and Axsäter and Marklund (2008). Early contributions include Simon (1971), Graves (1985) and Axsäter (1990), which consider systems with Poisson demand, complete backordering, FCFS allocation, and base-stock policies at all locations. Graves (1985) uses an exact approach similar to Simon (1971) for determining the probability distributions of the retailers inventory levels and the expected system costs. An accurate approximation is also presented. The analysis is based on determining the steady state probability distributions of the number of outstanding orders at each retailer by binomial disaggregation of the total number of outstanding orders. The approach is also commonly translated into disaggregation of the total number of warehouse backorders to obtain the distribution of warehouse backorders destined for each retailer (c.f., Axsäter 2006). Generalizing the method to handle compound Poisson demand is difficult and has so far not been done. The challenge is how to disaggregate the distribution of the total number of outstanding orders to specific retailers. 
Axsäter (1990) provides exact and computationally efficient recursive expressions for the system's expected inventory holding and backorder costs. His approach is based on tracking an arbitrary unit through the system, and determining the holding and backorder costs it incurs. The method does not involve the inventory level distributions at the retailers. Our work is related to both these approaches. As we are interested in the inventory levels in the system, we derive the exact probability distributions of warehouse backorders destined to each retailer. However, instead of disaggregating the total amount of backorders, we obtain these distributions by tracking what happens in the system backwards and forwards in time. The methodology of Axsäter (1990) has been extended in various ways to deal with more general divergent systems. For example, Axsäter (1993a, 1998) and Forsberg (1997) consider exact and approximate models with installation stock $(R, Q)$-policies and Poisson demand. In case of compound Poisson demand, Forsberg (1995) shows how to exactly calculate costs for base-stock policies at the retailers as weighted sums of the expected costs of $(\mathrm{S}-1, \mathrm{~S})$ systems with Poisson demand obtained from Axsäter (1990). Axsäter (1997) extends the model to echelon stock $(R, Q)$-policies (the echelon stock of an inventory location includes the stock at the location itself and at all downstream locations). In a parallel work, Chen and Zheng (1997) provide an alternative method for evaluating echelon-stock $(R, Q)$-policies that is exact for Poisson demand and approximate for compound Poisson demand. This approach is related to the disaggregation of warehouse backorders used by Simon (1971) and Graves (1985). Of particular interest for our work is Axsäter (2000), which provides exact analysis of the expected holding and backorder costs and the probability distributions for the inventory levels in a one-warehouse N-retailer system with installation stock $(R, Q)$-policies and compound Poisson demand. In the special case of no shipment consolidation and batch quantities equal to one at all retailers, our present work offers a different way to analyze the system in Axsäter (2000).

The literature on divergent continuous review systems also contains papers that investigate other warehouse ordering policies utilizing more detailed inventory and demand information. Marklund (2002) provides an exact analysis of a new type of service level policy at the warehouse (referred to as an $\left(\alpha_{0}, Q_{0}\right)$ policy) that uses real-time information about the individual inventory positions at all stockpoints. In a parallel work Moinzadeh (2002) investigates a generalized installation-stock $(R, Q)$ policy at the warehouse assuming identical retailers. Axsäter and Marklund (2008) derive a warehouse ordering policy that is optimal in the class of "position based" policies, which encompass all the policies discussed above. They also relax the FCFS assumption made in all previous models cited above. Marklund (2006) focuses on the use of advance order information, and provides exact and approximate analysis of warehouse reservation policies. These policies enable the warehouse to differentiate its service to the retailers through temporal allocation and prioritization. All the models 
above assume the use of partial deliveries, i.e., all stockpoints ship what is available as soon as possible.

Apart from the exact results mentioned above there exist a large number of approximations for analyzing one-warehouse-N-retailer inventory systems, (c.f., Axsäter (2003) for an overview). One of the most common approximation techniques is to replace the stochastic delay due to stockouts by its average value. This idea originates from the METRIC model by Sherbrooke (1968) and has been developed and adapted to many different settings in e.g. Sherbrooke (1986), Zipkin (2005 p.335), Andersson et al. (1998), Andersson and Marklund (2000), Axsäter (2003) and Berling and Marklund (2013, 2014). This group of approximations is related to our work as the average waiting time usually is obtained by dividing the average total amount of backorders at the central warehouse with the average total demand per time unit. In some special cases this renders the correct mean, but in general the average waiting time per demanded unit at a warehouse is different across the retailers. Our analysis of the backorders at the central warehouse can be used for determining the correct average waiting time for each retailer. In this respect our work is related to Kiesmüller et al. (2004), which focus on an approximation model involving the first two moments of the waiting time.

The time-based dispatching and use of fixed shipment intervals links our present work to the research on divergent periodic review systems. A major difference compared to the traditional periodic review literature (see, for example, Federgruen and Zipkin 1984, Jackson 1988, Federgruen 1993, Axsäter 1993b, Graves 1996, Houtum et al. 1996, Heijden et al. 1997, Diks and de Kok 1998,1999, Cachon 1999, Cachon and Fisher 2000, Axsäter et al. 2002, Özer 2003, Chu and Shen 2010, Marklund and Rosling 2012, Shang et al. 2014, and references therein) is that in our current model only shipments are made periodically, while inventory is reviewed and replenished continuously. Graves (1996), Axsäter (1993b), and Shang et al. (2014) have a closer relationship with our work because they assume a virtual (FCFS) allocation policy based on Poisson demand arrivals. This means that the inventory levels must be monitored continuously even though orders are generated periodically. Our model assumptions are less restrictive with respect to the compound Poisson demand and the $(R, Q)$-policy at the warehouse. A distinguishing feature of Shang et al. (2014) compared to Graves (1996) and Axsäter (1993b), is that not only the base-stock levels but also the reorder intervals are decision variables. An important contribution is that the authors are able to provide bounds that facilitate optimization of all these decision variables to minimize the total expected costs. The reorder intervals are related to the shipment intervals in our model.

Finally, there is a connection between our work and the body of literature that investigates different types of consolidation policies for outbound shipments in a single-echelon context. The focus in this literature is placed on a VMI supplier (typically with a negligible replenishment lead time) that receives 
orders from multiple retailers. The supplier wants to decide how and when to replenish and dispatch shipments in order to minimize its inventory and shipment costs. Examples from this literature include Çetinkaya and Lee (2000), Axsäter (2001), Çetinkaya and Bookbinder (2003), Çetinkaya et al. (2008), and Mutlu et al. (2010). In principle, these (and other) papers consider three types of consolidation strategies for Poisson or compound Poisson demand: (i) time-based consolidation policies where time dictates when shipments are dispatched, (ii) quantity-based policies where shipments are consolidated into fixed dispatch quantities, and (iii) time-and-quantity based policies where shipments leave either when a dispatch quantity is reached or when a certain time has passed since the last dispatch. None of these papers provide an exact analysis similar to our present work.

The remainder of this paper is organized as follows. Section 2, describes the detailed model assumptions and the associated total cost function for the single-item model. Section 3 presents exact analysis of the inventory level distributions as well as the backorder distributions at the central warehouse. Section 4 explains the optimization procedure. Section 6 extends the model formulation, cost analysis and optimization procedure to the multi-item case. Finally, Section 7 discusses possible extensions, future research directions and conclusions. The electronic companion of this paper containing all proofs and appendices is available together with the online version that can be found at http://or.journal.informs.org/.

\section{Problem Formulation Single-Item Model}

As explained above, the system under consideration is a centralized continuous review inventory system with one warehouse and $N$ non-identical retailers. Initially (in Sections 2, 3 and 4), we limit our attention to single-item systems. Extensions to multi-item systems are described in Section 6 . The warehouse replenishes its inventory from one or several outside suppliers with constant lead time $L_{0}$. The retailers order solely from the central warehouse (no lateral transshipments between retailers are allowed). All stockpoints use complete backordering and FCFS allocation of items to demands. Customer demand occurs at the retailers and follows stationary compound Poisson processes with discrete compounding distributions. Thus, customer orders at retailer i arrive according to a Poission process, and the size of each order is random with a discrete probability mass function (referred to as a compounding distribution). The demand processes across different retailers can be different but they are assumed to be mutually independent. An important rational for using the compound Poisson process is that it offers the flexibility to model highly variable customer demand processes with order size uncertainty. This is a characterizing feature of the industry example motivating this work, but it also applies to many other real world systems (e.g. Kapuscinski et al.(2004)). For practical examples 
of using compound Poisson processes to model real world demand we refer to, for example, Eaves (2002), Berling and Marklund (2013) and Lengu et al. (2014). Theoretically, the compound Poisson process is interesting because of its generality. For instance, it can be shown (Feller 1966, Axsäter 2006) that any demand process, where the cumulative demand follows a non-decreasing stochastic process with mutually independent increments (a common assumption in the inventory literature), can be represented by a limit of an appropriate sequence of compound Poisson processes.

Inventory control at the retailers is accomplished by base-stock policies with order-up-to level $S_{i}$ for retailer $i$. The use of base-stock policies is not an assumption per se, but a consequence of the assumption that POS (Point Of Sale) information is immediately transferred to the warehouse without any fixed costs associated with each transaction. Thus, there are no incentives to batch demand information from consecutive customers into consolidated orders. However, there are fixed costs associated with the transportation of physical products. These costs are considered centrally in the shipment consolidation of deliveries from the warehouse to the retailers over time and across groups of retailers sharing common delivery routes. In accordance with the referred literature on continuous review multi-echelon inventory systems all stockpoints apply a partial delivery policy. At the retailers, this means that a customer that orders more than what is on hand at the retailer will receive the units on hand and the remaining demand is backordered. Similarly at the warehouse, if only part of a retailer order (corresponding to a customer order) is on hand when a shipment is about to leave, the available units will be shipped immediately and the rest later. Partial deliveries are commonly used in practice (for instance, in the industry examples motivating this work) as it represents the fastest way to satisfy the customer demand. It dominates the obvious alternative of only delivering complete orders provided that there are no, or very small, costs associated with order splitting. This holds for example in a distribution system where there are only variable shipment costs (including picking, loading transporting and receiving) per unit handled. Or as in our case with periodic shipments, where there are variable and fixed shipment costs, but the latter are associated with the planned shipments, which are unaffected by order splitting. If splitting an order leads to an extra shipment associated with fixed costs, a partial delivery policy may be costly. Our method can be modified to deal with complete instead of partial deliveries analogously to the analysis in Howard and Stenius (2013).

The central warehouse uses a continuous review $\left(R_{0}, n Q_{0}\right)$ policy to control its inventory replenishments. This means that as soon as the (installation stock) inventory position (defined below) falls to or below $R_{0}$ an order of $n Q_{0}$ units is placed with an outside supplier/manufacturer. Here $n$ is the smallest integer that raises the inventory position above $R_{0}$. The batch quantity $Q_{0}$ is presumed 
to be given with the restriction that it must be a positive integer (in our motivating example it is typically determined by set up costs and restrictions at the outside supplier/manufacturer, but it can also be determined by a deterministic EOQ method, as suggested in Zheng 1992 and Axsäter 1996). Even though $Q_{0}$ is not a decision variable in our model, the presented method can of course be used repeatedly to evaluate different $Q_{0}$ options. Extensions to other replenishment policies at the central warehouse are discussed in Section 7. The use of continuous review base stock policies at the retailers implies that from a control perspective, there is no difference between using an installation stock policy and an echelon-stock policy at the warehouse. Thus the former concept is used in this paper.

The shipment consolidation policy for outbound warehouse deliveries means that there is a shipment leaving the central warehouse to retailer $i$ every $T_{i}$ time units. Henceforth, $T_{i}$ is referred to as the shipment interval of retailer $i$. Periodic shipments are often seen in practice as they facilitate planning of dispatching activities, such as picking, loading, transportation and receiving, and can therefore reduce the costs (see, for example, Gaur and Fisher 2004, and Kuhn and Sternbeck, 2013). In addition to consolidation of outbound deliveries to a single retailer over the shipment intervals, the model allows for consolidation of shipments to groups of retailers sharing the same delivery routes. This is done by synchronizing the shipment intervals (the lengths and moments of shipments) to all retailers on the same route, allowing the same vehicle(s) to service all retailers in that group with a joint shipment. Note that because of the FCFS allocation, synchronization of shipment intervals across retailer groups is of no relevance in our model. With slight abuse of notation we let $T_{k}$ denote the shipment interval to retailer group $k$. The retailer groups and the routing within the groups are taken as given input to our model and need to be determined exogenously. However, the model can of course be used to evaluate different groupings and routing alternatives. In the industry examples motivating this work, restrictions in transport capacity has not been considered an issue as it is bought from third party logistics providers/forwarders. Therefore no such restrictions are included in the model.

The shipment time from the warehouse to retailer $i, L_{i}$, includes not only handling activities such as picking, loading, transporting and receiving, but also the time to visit the preceding retailers on the same route. The replenishment lead times for retailer orders are stochastic and depend on the shipment times, the shipment intervals and the stock availability at the warehouse. Note that the latter two affect the delay at the warehouse before a unit is shipped. The replenishment lead time for the central warehouse, $L_{0}$, is assumed constant.

To further explain the sequence of events in the replenishment and delivery processes, consider the system as a customer arrives to retailer $i$ at time $t$, and demands $x$ units. The retailer then tries to satisfy the demand from its stock on-hand, and if there are less than $x$ units on hand, the shortage is 
backordered. These backorders are cleared in a FCFS sequence as forthcoming replenishments arrive from the warehouse. When the customer arrives at time $t$, the demand instantaneously translates into an order of $x$ units from retailer $i$ to the warehouse, which then reserves $x$ units for delivery to retailer $i$. If there is unreserved stock on-hand at the warehouse, these units are reserved first and are added to the reserved stock on-hand awaiting the next shipment to retailer $i, W_{i}$. If there is less than $x$ unreserved units on-hand, the remaining units are backordered (reserved units that are outstanding at time $t$ ). As replenishments arrive, the backorders are cleared in a FCFS sequence and the units are added to the reserved stock on-hand. When the next shipment is dispatched to retailer $i$, the reserved stock on-hand for this retailer, $W_{i}$, goes to zero as all these units leave the warehouse on a shipment to retailer $i$. Focusing on the replenishment process at the warehouse, the reservation of $x$ units at time $t$ lowers the warehouse's inventory position, defined as the outstanding orders + unreserved stock on-hand - backorders, by the same amount. When the inventory position falls to or below $R_{0}$, an order of $n Q_{0}$ units is placed with the outside supplier, bringing the inventory position back between $R_{0}+1$ and $R_{0}+Q_{0}$. Note that the reserved stock on-hand is excluded from the inventory position as these units cannot be used for satisfying future retailer orders. The reserved stock-on hand at the warehouse can for all practical purposes be seen as units on route to specific retailers.

Analogous to the inventory position above, the inventory level at the central warehouse, $I L_{0}$, is defined as the unreserved stock on-hand minus the backorders. Note, if $T_{i} \rightarrow 0 \forall i=1, \ldots, N$ and the warehouse uses partial deliveries to satisfy retailer orders, $I L_{0}$ represents the traditional installation-stock inventory level. The total stock on hand at the warehouse is the sum of the unreserved stock on-hand, $\max \left(I L_{0}, 0\right)$, and the reserved stock-on hand awaiting shipment to different retailers, $\sum_{i=1, \ldots, N} W_{i}$. The inventory level of retailer $i, I L_{i}$, is defined as the stock on hand minus the amount of backorders at this retailer. The inventory position at retailer $i$ is the inventory level plus all outstanding orders, and it is kept at the base stock level $S_{i}$ at all times.

The FCFS allocation policy is commonly used in practice. It is generally considered as a "fair" allocation policy that is easy to implement. In the current model, it also provides an incentive for the retailers to share their point-of-sale information immediately with the warehouse. However, it is clearly not an optimal allocation policy. Inventory allocation in divergent multi-echelon systems with stochastic demand is an inherently difficult problem, and no general optimality results exist. FCFS allocation allows tractability and is a standard assumption in the continuous review literature. To our knowledge relaxations are only analyzed exactly in Marklund (2006) and Axsäter and Marklund (2008) both assuming Poisson demand. FCFS allocation is also used in numerous periodic review models of divergent systems starting with Graves (1996) coining the term virtual allocation. Turning 
to our current model setting, Howard and Marklund (2011) and Howard (2013) have investigated the performance of FCFS allocation in comparison to four state dependent myopic allocation policies (two in each paper) under the assumption of Poisson demand. The two policies in Howard (2013) have a performance guarantee over FCFS. In each paper, the two policies analyzed are based on postponing the allocation to the moment of shipment, and the moment of delivery respectively. The numerical results obtained by simulation show that when allocation is performed at the moment of delivery, the cost savings of using the more sophisticated policies instead of FCFS can be significant: on average 5.6\% in Howard and Marklund (2011) and 6.7\% in Howard (2013). Allocation at moment of delivery is for practical reasons very challenging to implement and is associated with additional costs for inventory handling and IT, which are not included in the analysis. When allocation is performed at the moment of shipment, which is much cheaper and easier to implement, the savings are quite small: $1.6 \%$ and $2.4 \%$ respectively. The compound Poisson assumption in our present work implies larger demand variability than in the studied models with Poisson demand. However, there are no indications in these studies that a larger standard deviation to mean ratio of the demand per time unit (i.e. a lower customer arrival rate) has any significant impact on the FCFS performance. Still, further investigation of the performance of FCFS allocation for compound Poisson demand is an interesting venue for future research. In a separate study, Graves (1996) finds a lower bound on the costs in a distribution system with periodic replenishments for any allocation policy. A numerical study indicates that the cost increase of using FCFS (referred to as virtual allocation) is modest. Thus our choice of FCFS allocation is motivated by its wide spread use in practice and in theory, but also by its analytical tractability and seemingly good performance.

We use the following notation to express the system stock levels and demand structure:

$N$ : Number of retailers

$K$ : Number of retailer groups $(\leq N)$

$N_{k}$ : Set of retailers belonging to retailer group $k$

$\lambda_{i}$ : Arrival rate of customers at retailer $i$

$Y_{i}$ : Number of units demanded by an arbitrary customer at retailer $i$, stochastic variable, $\mu_{i}=E\left[Y_{i}\right]$ (Note by assumption $Y_{i}>0$ and thus $P\left(Y_{i}=0\right)=0$.)

$\lambda_{0}$ : Arrival rate of retailer orders at the warehouse

$Y_{0}$ : Number of units in a retailer order at the warehouse, stochastic variable

$\lambda_{i c}$ : Arrival rate of retailer orders at the warehouse excluding orders from retailer $i$

$Y_{i^{c}}$ : Number of units in a retailer order at the warehouse excluding orders from retailer $i$, stochastic variable 
$D_{i}\left(t_{1}, t_{2}\right)$ : Customer demand at retailer $i$ in the time interval $\left(t_{1}, t_{2}\right]$, where $t_{1} \leq t_{2}$

$D_{i}(x)$ : Total customer demand at retailer $i$ during $x$ units of time (for simplicity $D_{i} \equiv D_{i}(1)$ )

$D_{0}(x)$ : Aggregate demand at the warehouse during $x$ units of time $\left(D_{0} \equiv D_{0}(1)\right)$

S: Vector of retailer order-up-to levels $=\left(S_{1}, \ldots, S_{N}\right)$

$T_{i}$ : Shipment interval to retailer $i\left(=T_{k}\right.$ for all retailers $\left.i \in N_{k}\right)$

$\mathbf{T}$ : Vector of shipment intervals to all retailers $=\left(T_{1}, \ldots, T_{N}\right)$

$I L_{i}(t)$ : Inventory level at retailer $i$ at time $t$ (= stock on hand - backorders)

$W_{i}(t)$ : Reserved stock on hand at the warehouse destined for retailer $i$ at time $t$

$I L_{0}(t)$ : Inventory level at the warehouse at time $t$ (= unreserved stock on-hand - backorders)

$I P_{0}(t)$ : Inventory position of the warehouse at time $t\left(I L_{0}(t)+\right.$ outstanding orders $)$

$x^{+}=\max (x, 0)$

$x^{-}=\max (-x, 0)$

As the retailer order processes are identical to the customer demand processes, $\lambda_{0}=\sum_{i=1}^{N} \lambda_{i}$ and $Y_{0}=\sum_{i=1}^{N} \chi_{i} Y_{i}$, where $\chi_{i}$ is an indicator function that is 1 if a given order is from retailer $i$ and 0 otherwise. The probability that a given order emanates from retailer $i$ is $P\left\{\chi_{i}=1, \chi_{j}=0 \forall j \neq\right.$ $i\}=\frac{\lambda_{i}}{\lambda_{0}}$ for all $i \in N$. Analogously $\lambda_{i^{c}}=\sum_{j \in N \backslash i} \lambda_{j}$ and $Y_{i^{c}}=\sum_{j \in N \backslash i} \chi_{j} Y_{j}$. Based on the definitions above, the probability mass function (pmf) of the demand during $x$ time units at retailer $i$ is

$$
P\left\{D_{i}(x)=y\right\}=\sum_{n=1}^{y} \frac{\left(\lambda_{i} x\right)^{n}}{n !} e^{-\lambda_{i} x} P\left\{Y_{i}^{n}=y\right\}, \quad y \geq 0
$$

where $Y_{i}^{n}$ represents the total amount of units demanded by $n$ customer orders arriving to retailer $i$. More precisely, $Y_{i}^{n}$ is the sum of $n$ i.i.d. customer orders, $Y_{i}$, and the pmf of $Y_{i}^{n}$ is determined by the $n$-fold convolution of the pmf of $Y_{i} . P\left\{D_{0}(x)=y\right\}$ and $Y_{0}^{n}$ are determined analogously.

With respect to cost parameters, the model considers inventory holding costs per unit and time unit at all inventory locations, denoted $h_{i}$ for $i=0,1, \ldots, N$, and backorder costs per unit and time unit at all retailers, denoted $\beta_{i}$ for $i=1, \ldots, N$. Moreover, a shipment cost of $\omega_{k}$ is incurred for each scheduled shipment leaving the central warehouse for retailer group $k$. This reflects, for example, the use of a third party logistics provider or forwarder (as in our motivating industry example) with a fixed cost as part of the contract. Note that $\omega_{k}$ should capture the fixed costs for all retailers in group $k$, and is incurred even if there are no units to ship. There are also per unit costs for shipping to a specific retailer. However, due to the complete backordering, all demanded units will eventually be shipped. Thus the shipment costs per unit do not affected the optimization and are therefore excluded from the analysis. 
The objective is to minimize the long-run average total cost, $T C\left(R_{0}, \mathbf{S}, \mathbf{T}\right)$, in (2) with respect to $R_{0}, \mathbf{S}$ and $\mathbf{T}$. This total cost consists of: (i) The expected warehouse holding cost per time unit, $h_{0}\left(E\left[I L_{0}^{+}\right]+\sum_{i=1, \ldots, N} E\left[W_{i}\right]\right)$, (ii) the expected holding and backorder costs per time unit at retailer $i, h_{i} E\left[I L_{i}^{+}\right]$and $\beta_{i} E\left[I L_{i}^{-}\right]$, and (iii) the expected shipment cost per time unit $S C(\mathbf{T})=\sum_{k=1, \ldots, K} \frac{\omega_{k}}{T_{k}}$. Note that the latter is independent of $R_{0}$ and $\mathbf{S}$ and only depends on the shipment intervals.

$T C\left(R_{0}, \mathbf{S}, \mathbf{T}\right) \quad=h_{0}\left(E\left[I L_{0}^{+}\right]+\sum_{i=1, \ldots, N} E\left[W_{i}\right]\right)+\sum_{i=1, \ldots, N}\left(h_{i} E\left[I L_{i}^{+}\right]+\beta_{i} E\left[I L_{i}^{-}\right]\right)+\sum_{k=1, \ldots, K} \frac{\omega_{k}}{T_{k}}$.

In a system with fill rate constraints, the objective is to minimize the total costs while meeting the fill rate constraint for each retailer. In this case there will be no backorder costs at the retailers and the total cost function will only consist of holding and shipment costs, that is, the term $\beta_{i} E\left[I L_{i}^{-}\right]$ disappears from (2). The fill rate is defined as the portion of the total demand that can be satisfied from stock on hand. How to analyze the fill rates is described at the end of Section 3.2.

\section{Analysis}

In this section, we provide an exact analysis of the probability mass function of the inventory levels and the expected inventory holding and backorder costs for the single-item system. The analysis is valid for any combination of the decision variables $R_{0}, \mathbf{S}$ and $\mathbf{T}$. In Section 3.1 we determine the average stock on hand (both unreserved and reserved) at the central warehouse. Section 3.2 explains how to compute the average stock on hand, backorders and fill rates at each retailer $i$. This analysis assumes that the probability mass functions of the backorders at the central warehouse, designated to retailer $i \forall i \in N$ are known. Section 3.3 provides an exact approach for determining these probabilities. All proofs are deferred to the online Appendix B, and an illustrative numerical example is available in the online Appendix C. All online appendices are found in the electronic companion.

\subsection{The Stock on Hand at the Central Warehouse}

As explained above, the stock on hand at the central warehouse consist of unreserved stock on hand, $I L_{0}^{+}$, and reserved stock on hand for each retailer $i, W_{i}$. Note that the shipment consolidation policy has no impact on the unreserved stock on hand or the backorders at the central warehouse. The inventory level at the central warehouse, $I L_{0}$, (per definition excluding the reserved stock on hand) can therefore be analyzed as a single-echelon system without shipment consolidation. Thus, in steady state $I L_{0}=I P_{0}-D_{0}\left(L_{0}\right)$. Moreover, because of the centralized access to POS information (manifested 
by the continuous review base-stock policies at the retailers) the order process at the warehouse is a superposition of the compound Poisson demand process at the retailers. Assuming that not all customer demand sizes are multiples of some integer larger than one, the inventory position in steady state is uniform on $\left[R_{0}+1, R_{0}+Q_{0}\right]$ (see Axsäter 2006 p.88). It follows that

$$
E\left[I L_{0}^{+}\right]=\frac{1}{Q_{0}} \sum_{I P_{0}=R_{0}+1}^{R_{0}+Q_{0}} E\left[\left(I P_{0}-D_{0}\left(L_{0}\right)\right)^{+}\right] .
$$

The expected reserved stock on hand destined for retailer $i, W_{i}$, is given in Proposition 1.

Proposition 1. The expected stock on hand at the central warehouse reserved for retailer $i$ is

$$
E\left[W_{i}\right]=\frac{1}{2} \lambda_{i} \mu_{i} T_{i}
$$

\subsection{Inventory Levels at the Retailers}

To derive the probability mass function for the inventory level at a given retailer, consider a shipment destined for this retailer (group) that leaves the warehouse at time $t_{0}$. This shipment arrives at retailer $i$ at time $t_{0}+L_{i}$ and the next shipment will arrive at $t_{0}+L_{i}+T_{i}$. We call this recurring time interval, $\left(t_{0}+L_{i}, t_{0}+L_{i}+T_{i}\right]$, a replenishment cycle for retailer $i$ (see Figure 1$)$.

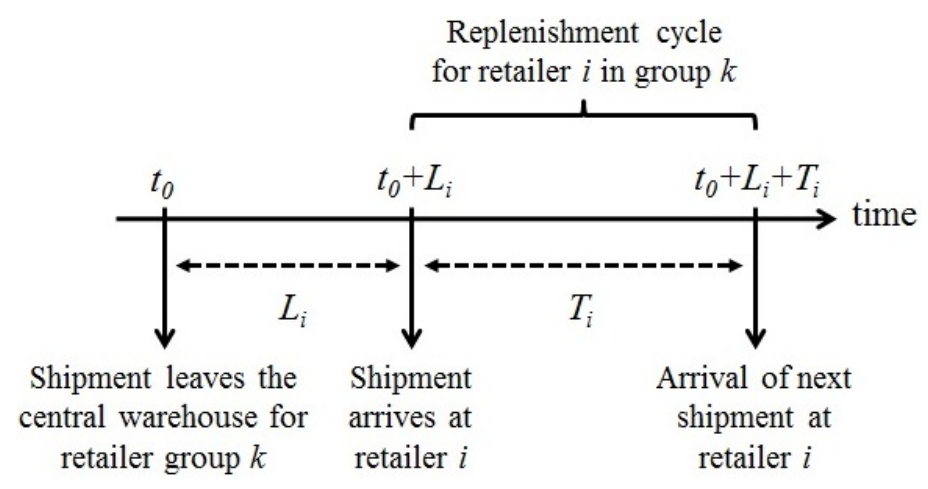

Figure 1: Replenishment cycle for retailer $i$

The inventory level for retailer $i$ at $t_{0}+L_{i}+t\left(0<t \leq T_{i}\right)$ is determined by the inventory level at $t_{0}+L_{i}$, just after the considered shipment has arrived, and the demand in $\left(t_{0}+L_{i}, t_{0}+L_{i}+t\right]$. Defining $B_{i}\left(t_{0}\right)$ as the number of backordered units for retailer $i$ at the central warehouse at $t_{0}$,

$$
\begin{aligned}
I L_{i}\left(t_{0}+L_{i}+t\right) & =I L_{i}\left(t_{0}+L_{i}\right)-D_{i}\left(t_{0}+L_{i}, t_{0}+L_{i}+t\right) \\
& =S_{i}-B_{i}\left(t_{0}\right)-D_{i}\left(t_{0}, t_{0}+L_{i}+t\right), \quad 0<t \leq T_{i} .
\end{aligned}
$$

Note that $B_{i}\left(t_{0}\right)$ and $D_{i}\left(t_{0}, t_{0}+L_{i}+t\right)$ are independent since $B_{i}\left(t_{0}\right)$ depends on the demand before 
time $t_{0}$. The pmf of the inventory level of retailer $i$ at $t_{0}+L_{i}+t$ can then be obtained as

$$
P\left\{I L_{i}\left(t_{0}+L_{i}+t\right)=j\right\}=\sum_{r=0}^{S_{i}-j} P\left\{B_{i}\left(t_{0}\right)=r\right\} P\left\{D_{i}\left(L_{i}+t\right)=S_{i}-j-r\right\}, \quad j \leq S_{i}
$$

The challenging part in (6) is to determine the probabilities $P\left\{B_{i}\left(t_{0}\right)=r\right\}$. Section 3.3 explains how this can be done. For now we assume that these probabilities are known and focus on determining the expected stock on hand, and the expected backorders at retailer $i$. The former is obtained as the expected stock on hand during a replenishment cycle divided by the cycle length, see (7).

$$
\begin{aligned}
E\left[I L_{i}^{+}\right] & =\frac{1}{T_{i}} \int_{0}^{T_{i}} E\left[I L_{i}\left(t_{0}+L_{i}+x\right)^{+}\right] d x \\
& =\frac{1}{T_{i}} \sum_{j=1}^{S_{i}} j \int_{0}^{T_{i}} P\left\{I L_{i}\left(t_{0}+L_{i}+x\right)=j\right\} d x \\
& =\frac{1}{T_{i}} \sum_{j=1}^{S_{i}} \sum_{r=0}^{S_{i}-j} j P\left\{B_{i}\left(t_{0}\right)=r\right\} \int_{0}^{T_{i}} P\left\{D_{i}\left(L_{i}+x\right)=S_{i}-j-r\right\} d x
\end{aligned}
$$

The expected inventory level can be determined analogously,

$$
E\left[I L_{i}\right]=S_{i}-E\left[B_{i}\left(t_{0}\right)\right]-\lambda_{i} \mu_{i}\left(L_{i}+\frac{T_{i}}{2}\right)
$$

Based on these results, the expected number of backorders at retailer $i$ can be obtained from (9).

$$
E\left[I L_{i}^{-}\right]=E\left[I L_{i}^{+}\right]-E\left[I L_{i}\right]
$$

The fill rate for retailer $i$, denoted by $\gamma_{i}$, can then be determined from (10). This expression follows from analysis of single-echelon systems with compound Poisson demand, see, for example, Axsäter (2006). The difference between the stock on hand at the beginning and at the end of the replenishment cycle renders the amount of demand satisfied directly from stock. This amount is divided by the total expected demand during a cycle. Recall that in systems with fill rate constraints we assume that there are no backorder costs, and the total cost consists of the sum of the holding and shipment costs.

$$
\gamma_{i}=\frac{E\left[I L_{i}\left(t_{0}+L_{i}\right)^{+}\right]-E\left[\left(I L_{i}\left(t_{0}+L_{i}\right)-D\left(t_{0}+L_{i}, t_{0}+L_{i}+T_{i}\right)\right)^{+}\right]}{\lambda_{i} \mu_{i} T_{i}}
$$




\subsection{Distribution of the Warehouse Backorders}

Consider the central warehouse at time $t_{0}$ when a shipment is leaving the central warehouse. We are interested in the pmf of $B_{i}\left(t_{0}\right)$, the number of backordered units destined for retailer $i$. The analysis focuses on the inventory level at the warehouse, $I L_{0}$ (where all the backorders at the central warehouse are included). The backorders at time $t_{0}$ depend on the inventory position a replenishment lead time earlier, $I P_{0}\left(t_{0}-L_{0}\right)$, and the demand during the lead time. Since the inventory position in steady state is uniformly distributed on $\left[R_{0}+1, R_{0}+Q_{0}\right]$, the pmf of $B_{i}\left(t_{0}\right)$ can be obtained as

$$
P\left\{B_{i}\left(t_{0}\right)=r\right\}=\frac{1}{Q_{0}} \sum_{S_{0}=R_{0}+1}^{R_{0}+Q_{0}} P\left\{B_{i}\left(t_{0}\right)=r \mid I P_{0}\left(t_{0}-L_{0}\right)=S_{0}\right\}
$$

The remaining analysis focuses on deriving expressions for $P\left\{B_{i}\left(t_{0}\right)=r \mid I P_{0}\left(t_{0}-L_{0}\right)=S_{0}\right\}$ and is divided in two cases: $S_{0}>0$ (in Section 3.3.1) and $S_{0} \leq 0$ (in Section 3.3.2).

\subsubsection{The Case of $S_{0}>0$}

Note first that backorders at time $t_{0}$ can only occur when the demand during the replenishment lead time, $D_{0}\left(L_{0}\right)$, is larger than $S_{0}$. Thus, the total amount of units backordered at time $t_{0}, B_{0}\left(t_{0}\right)$, is

$$
B_{0}\left(t_{0}\right)=\left(D_{0}\left(L_{0}\right)-S_{0}\right)^{+}
$$

Because of the FCFS policy, it will always be the last units demanded in $\left(t_{0}-L_{0}, t_{0}\right]$ that are backordered. To track these units, we therefore study the retailer orders during $\left(t_{0}-L_{0}, t_{0}\right]$. We define:

$\Phi_{0}$ : Total number of retailer orders arriving to the central warehouse (i.e., number of customers arriving to the system) during $\left(t_{0}-L_{0}, t_{0}\right]$.

$\Psi^{n}$ : The nominal inventory position $=$ the inventory position at the central warehouse at time $t_{0}-L_{0}$ minus the aggregate demand of the $n$ first retailer orders after time $t_{0}-L_{0}, Y_{0}^{n}$,

$$
\Psi^{n}=S_{0}-Y_{0}^{n}
$$

The nominal inventory position helps us to track which retailer orders are backordered at time $t_{0}$. Note that $\Psi^{0}=S_{0}$ and $\Psi^{\Phi_{0}}=I L_{0}\left(t_{0}\right)$, but for all other values $0<n<\Phi_{0}, \Psi^{n}$ can neither be seen as the inventory position nor the inventory level.

For the analysis we divide the backorders in two categories; partial backorders, $\hat{B}_{0}\left(t_{0}\right)$, and complete backorders, $\check{B}_{0}\left(t_{0}\right)$, such that $B_{0}\left(t_{0}\right)=\hat{B}_{0}\left(t_{0}\right)+\check{B}_{0}\left(t_{0}\right)$. The partial backorders (at time $\left.t_{0}\right)$ result 
from a retailer order that brings the nominal inventory position from a strictly positive value to a non-positive value. Hence, there can be at most one retailer order that is partially backordered, but it may involve many units that are all referred to as partial backorders. All orders after the nominal inventory position has reached zero will be completely backordered and all these units are referred to as complete backorders, see Figure 2(a).

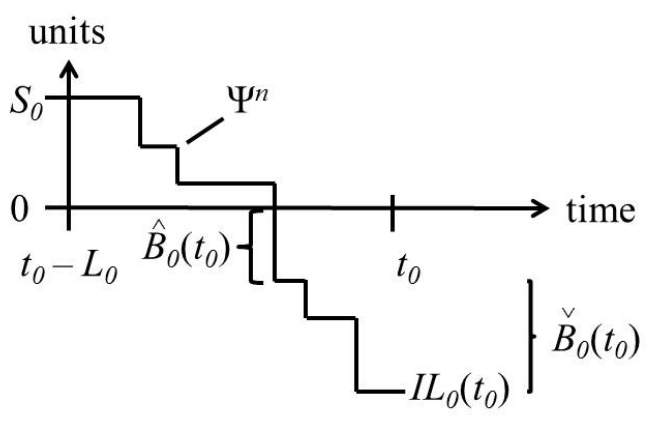

(a) $S_{0}>0$

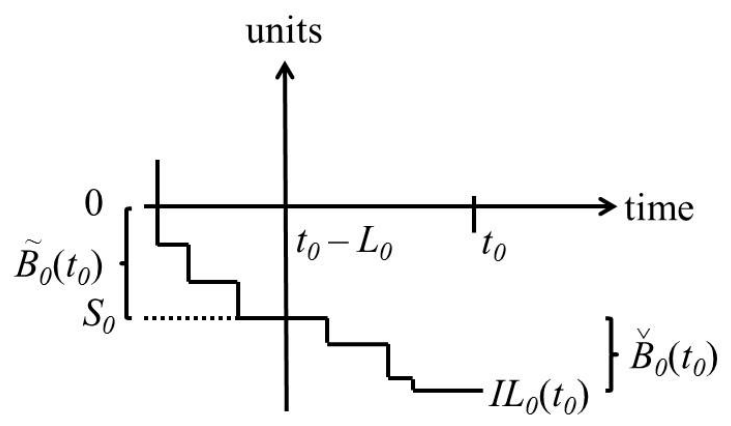

(b) $S_{0} \leq 0$

Figure 2: Illustration of two possible sample paths for the nominal inventory position, $\Psi^{n}$, at the central warehouse and the associated number of partial and complete backorders

Starting with the analysis of the partial backorders for retailer $i$ we define

$\hat{B}_{i}^{n}\left(t_{0}\right)$ : Number of partial backorders for retailer $i$ at $t_{0}$, when the $n^{\text {th }}$ retailer order after $t_{0}-L_{0}$ brings the nominal inventory position to a non-positive value.

Lemma 1. The probability of $u$ partial backorders for retailer $i$ at time $t_{0}$, when the $n^{\text {th }}$ customer order after $t_{0}-L_{0}$ brings the nominal inventory position to 0 or below, $P\left\{\hat{B}_{i}^{n}\left(t_{0}\right)=u\right\}$, is for $u>0$

$$
P\left\{\hat{B_{i}^{n}}\left(t_{0}\right)=u\right\}=P\left\{\Phi_{0} \geq n\right\} \sum_{x=1}^{S_{0}-n+1} P\left\{\Psi^{n-1}=x\right\} \frac{\lambda_{i}}{\lambda_{0}} P\left\{Y_{i}=u+x\right\},
$$

and for $u=0$

$$
P\left\{\hat{B_{i}^{n}}\left(t_{0}\right)=0\right\}=P\left\{\Phi_{0} \geq n\right\} \sum_{x=1}^{S_{0}-n+1} P\left\{\Psi^{n-1}=x\right\}\left(\frac{\lambda_{i}}{\lambda_{0}} P\left\{Y_{i}=x\right\}+\frac{\lambda_{i^{c}}}{\lambda_{0}} P\left\{Y_{i^{c}} \geq x\right\}\right)
$$

Turning to the retailer orders that are completely backordered, we know that after the nominal inventory position has reached zero for the $n^{\text {th }}$ retailer order, all subsequent orders are classified as complete backorders. Consequently, the distribution of the complete backorders is dependent on $n$.

$\check{B}_{i, n}\left(t_{0}\right)$ : Number of complete backorders for retailer $i$ at $t_{0}$, given that the nominal inventory position is taken from a positive to a non-positive value by the $n^{\text {th }}$ retailer order after time $t_{0}-L_{0}$ and $\Phi_{0} \geq n$. 
$\check{\Phi}_{i, n}$ : Number of orders from retailer $i$ before $t_{0}$ but after the $n^{\text {th }}$ retailer order has arrived to the warehouse, given $\Phi_{0} \geq n$.

$Z_{i}^{m}$ : number of orders from retailer $i$ given $m$ orders in total.

Note that $Z_{i}^{m}$ is binomially distributed due to the Poisson arrivals of customer orders:

$$
P\left\{Z_{i}^{m}=a\right\}=\left(\begin{array}{c}
m \\
a
\end{array}\right)\left(\frac{\lambda_{i}}{\lambda_{0}}\right)^{a}\left(\frac{\lambda_{0}-\lambda_{i}}{\lambda_{0}}\right)^{m-a} .
$$

Lemma 2. The probability of $v$ complete backorders for retailer $i$ given that the $n^{\text {th }}$ retailer order after $t_{0}-L_{0}$ brings $\Psi^{n}$ to a non-positive value, $P\left\{\check{B}_{i, n}\left(t_{0}\right)=v\right\}$, can be obtained as

$$
P\left\{\check{B}_{i, n}\left(t_{0}\right)=v\right\}=\sum_{a=1}^{v} P\left\{\check{\Phi}_{i, n}=a\right\} P\left\{Y_{i}^{a}=v\right\}
$$

where

$$
P\left\{\check{\Phi}_{i, n}=a\right\}=\sum_{m=a}^{\infty} \frac{P\left\{\Phi_{0}=n+m\right\}}{P\left\{\Phi_{0} \geq n\right\}} P\left\{Z_{i}^{m}=a\right\}
$$

Denoting the probability that the nominal inventory position never reaches zero by $p_{0}$, we have

$$
p_{0}=\sum_{n=0}^{S_{0}-1} P\left\{\Phi_{0}=n\right\} P\left\{\Psi^{n}>0\right\}
$$

The pmf of the number of backordered units for $S_{0}>0$ can now be obtained from Proposition 2 .

Proposition 2. The probability that the central warehouse has $r$ backordered units allocated to retailer $i$ at $t_{0}$ when the inventory position is $S_{0}>0$ can be obtained for $r>0$ as

$$
P\left\{B_{i}\left(t_{0}\right)=r \mid I P_{0}\left(t_{0}-L_{0}\right)=S_{0}\right\}=\sum_{n=1}^{S_{0}} \sum_{u=0}^{r} P\left\{\hat{B}_{i}^{n}\left(t_{0}\right)=u\right\} P\left\{\check{B}_{i, n}\left(t_{0}\right)=r-u\right\}
$$

and for $r=0$ as

$$
P\left\{B_{i}\left(t_{0}\right)=0 \mid I P_{0}\left(t_{0}-L_{0}\right)=S_{0}\right\}=p_{0}+\sum_{n=1}^{S_{0}} P\left\{\hat{B}_{i}^{n}\left(t_{0}\right)=0\right\} P\left\{\check{B}_{i, n}\left(t_{0}\right)=0\right\} .
$$

Remark. The expected amount of backorders is not always proportional to the demand per time unit resulting in different average waiting times per unit due to stockouts across the retailers. The expected amount of complete backorders are proportional to the demand per time unit, but this is not always true for the expected amount of partial backorders. 


\subsubsection{The Case of $S_{0} \leq 0$}

When the initial inventory position is less than or equal to zero, all units ordered during the time interval $\left(t_{0}-L_{0}, t_{0}\right]$ will be completely backordered at time $t_{0}$. We denote this part of the backorders $\check{B}_{0}\left(t_{0}\right)$ and the units ordered by retailer $i, \check{B}_{i}\left(t_{0}\right)$. In addition to this, the last $-S_{0}$ units ordered before time $t_{0}-L_{0}$ will also be backordered at time $t_{0}$. We denote this part of the backorders as $\tilde{B}_{0}\left(t_{0}\right)$ and the units ordered by retailer $i \tilde{B}_{i}\left(t_{0}\right)$, see Figure $2(\mathrm{~b})$. It follows that $B_{0}\left(t_{0}\right)=\check{B}_{0}\left(t_{0}\right)+\tilde{B}_{0}\left(t_{0}\right)$, $B_{i}\left(t_{0}\right)=\check{B}_{i}\left(t_{0}\right)+\tilde{B}_{i}\left(t_{0}\right)$ and $B_{0}\left(t_{0}\right)=\sum_{i=1}^{N} B_{i}\left(t_{0}\right)$. Note that $\check{B}_{0}\left(t_{0}\right)$ only consists of units from completely backordered retailer orders (ordered after $\left.t_{0}-L_{0}\right)$, while $\tilde{B}_{0}\left(t_{0}\right)\left(=-S_{0}\right)$ can consist of units both from completely backordered retailer orders, and from a partially backordered retailer order. As $\tilde{B}_{i}\left(t_{0}\right)$ depends on the demand before time $t_{0}-L_{0}$, and $\check{B}_{i}\left(t_{0}\right)$ depends on the demand during $\left(t_{0}-L_{0}, t_{0}\right]$, they are independent. The probability of $r$ units backordered at the warehouse for retailer $i$ at time $t_{0}$, when $S_{0} \leq 0$ is thus

$$
P\left\{B_{i}\left(t_{0}\right)=r \mid I P_{0}\left(t_{0}-L_{0}\right)=S_{0}\right\}=\sum_{u=0}^{\min \left(r,-S_{0}\right)} P\left\{\tilde{B}_{i}\left(t_{0}\right)=u\right\} P\left\{\check{B}_{i}\left(t_{0}\right)=r-u\right\}, \quad S_{0} \leq 0 .
$$

Because all units ordered in time interval $\left(t_{0}-L_{0}, t_{0}\right]$ will be completely backordered, the probability that $v$ units are backordered for retailer $i$ at $t_{0}$ is simply

$$
P\left\{\check{B}_{i}\left(t_{0}\right)=v\right\}=P\left\{D_{i}\left(t_{0}-L_{0}, t_{0}\right)=v\right\}
$$

Turning to the analysis of $\tilde{B}_{i}\left(t_{0}\right)$, note first that for $S_{0}=0$, there can be no backorders ordered before $t_{0}-L_{0}$. Consequently $\tilde{B}_{i}\left(t_{0}\right)=0 \forall i$, which means that $P\left\{\tilde{B}_{i}\left(t_{0}\right)=0\right\}=1 \forall i$ and $(22)$ simplifies to $P\left\{B_{i}\left(t_{0}\right)=r \mid I P_{0}\left(t_{0}-L_{0}\right)=0\right\}=P\left\{\check{B}_{i}\left(t_{0}\right)=r\right\}$. In order to determine $\tilde{B}_{i}\left(t_{0}\right)$ for $S_{0}<0$ we study the system backwards in time from $t_{0}-L_{0}$. We define

$\Psi^{-m}$ : Nominal inventory position before $t_{0}-L_{0}$, defined as the inventory position at time $t_{0}-L_{0}$ plus the accumulated demand of the last $m$ retailer orders before $t_{0}-L_{0}$.

$\tilde{B}_{i}^{-m}\left(t_{0}\right)$ : Backordered units to retailer $i$ at $t_{0}$ ordered before $t_{0}-L_{0}$, when the nominal inventory position reaches a non-negative value by the occurrence of the $m^{t h}$ retailer order before $t_{0}-L_{0}$ (i.e., counting backwards from $t_{0}-L_{0}$, the $m^{\text {th }}$ retailer order is the first order that is backordered; either completely or partially).

$V_{i}^{m}$ : The number of units ordered by retailer $i$, given that a total of $m$ retailer orders have occurred. 
Because the nominal inventory position can reach zero only once, $\tilde{B}_{i}^{-m}\left(t_{0}\right)$ for $m \in\left[1,-S_{0}\right]$ represent mutually exclusive events. Thus, we get

$$
P\left\{\tilde{B}_{i}\left(t_{0}\right)=u\right\}= \begin{cases}\sum_{m=1}^{S_{0}} P\left\{\tilde{B}_{i}^{-m}\left(t_{0}\right)=u\right\}, & S_{0}<0 \\ 1, & u=0 \text { and } S_{0}=0 \\ 0, & \text { otherwise }\end{cases}
$$

where $P\left\{\tilde{B}_{i}^{-m}\left(t_{0}\right)=u\right\}$ can be determined from Lemma 3 .

Lemma 3. The probability for $u$ backordered units at $t_{0}$, ordered by retailer $i$ before time $t_{0}-L_{0}$, when the $m^{\text {th }}$ customer order before $t_{0}-L_{0}$ is the first to be backordered, is

$$
\begin{aligned}
P\left\{\tilde{B}_{i}^{-m}=u\right\}=\sum_{x=S_{0}+m-1}^{-1} \quad & \frac{\lambda_{i^{c}}}{\lambda_{0}} P\left\{Y_{i^{c}} \geq-x\right\} P\left\{V_{i}^{m-1}=u \text { and } \Psi^{-(m-1)}=x\right\} \\
+\quad & \frac{\lambda_{i}}{\lambda_{0}} P\left\{Y_{i} \geq-x\right\} P\left\{V_{i}^{m-1}=u+x \text { and } \Psi^{-(m-1)}=x\right\},
\end{aligned}
$$

where

$$
\begin{aligned}
& P\left\{V_{i}^{m-1}=u \text { and } \Psi^{-(m-1)}=x\right\}=\sum_{a=0}^{m-1} P\left\{Z_{i}^{m-1}=a\right\} P\left\{Y_{i}^{a}=u\right\} P\left\{Y_{i^{c}}^{m-1-a}=x-S_{0}-u\right\} . \\
& P\left\{B_{i}\left(t_{0}\right)=r \mid I P_{0}\left(t_{0}-L_{0}\right)=S_{0}\right\} \text { for } S_{0} \leq 0 \text { follows from }(22),(23),(24) \text { and Lemma } 3 .
\end{aligned}
$$

\section{Optimization}

In this Section we present a method for optimizing the system parameters $R_{0}, \mathbf{S}$ and $\mathbf{T}$ both in systems with backorder costs, and in systems with fill rate constraints. We assume, as before, that the order quantity $Q_{0}$ is given by the outside supplier/manufacturer. The method is explained for the single-item case and is extended to the multi-item case in Section 6. The objective is to minimize the total cost function (2), or the sum of expected shipment and holding costs subject to fill rate constraints. We know from Section 3.1 that the stock on hand, and therefore also the holding costs, at the warehouse can be separated into unreserved stock on hand, $I L_{0}^{+}$, which depends on $R_{0}$ but is independent of $\mathbf{T}$ and $\mathbf{S}$, and the reserved stock on hand that depends on $\mathbf{T}$ but is independent of $R_{0}$ and $\mathbf{S}$. We define the costs directly related to retailer group $k$ (including the holding cost of the 
reserved stock on hand going to the retailers in the retailer group) as

$$
T C_{k}\left(R_{0}, \mathbf{S}, T_{k}\right)=\frac{\omega_{k}}{T_{k}}+\sum_{i \in N_{k}} h_{0} E\left[W_{i}\right]+h_{i} E\left[I L_{i}^{+}\right]+\beta_{i} E\left[I L_{i}^{-}\right]
$$

Recall that, with slight abuse of notation, we let $T_{k}$ denote the shipment interval for all retailers in retailer group $\mathrm{k}\left(T_{k}=T_{i} \forall i \in N_{k}\right)$. The total cost function can thus be expressed as

$$
T C\left(R_{0}, \mathbf{S}, \mathbf{T}\right)=h_{0} E\left[I L_{0}^{+}\right]+\sum_{k \in K} T C_{k}\left(R_{0}, \mathbf{S}, T_{k}\right)
$$

The total cost function is not jointly convex in $R_{0}, \mathbf{S}$ and $\mathbf{T}$ which can be shown by example. Thus, the proposed optimization method is based on bounding $R_{0}$ and $T_{k} \forall k$, using Proposition 4 and Proposition 5 respectively, and searching this bounded region using the convexity property of the retailer order-up-to levels specified in Proposition 3. The approach for obtaining the bounds in Propositions 4 and 5 is to assert when further changes of the considered decision variable can no longer decrease the total cost below the lowest cost known so far, $\overline{T C}$.

Proposition 3. For fixed $R_{0}$ and $\boldsymbol{T}$ the total cost function $T C\left(R_{0}, \boldsymbol{S}, \boldsymbol{T}\right)$ is convex and separable in the retailer order-up-to levels $\boldsymbol{S}$.

\subsection{Optimization procedure}

The first step in the optimization procedure is to determine lower bounds for the optimal total costs directly related to each retailer group $k \in K, T C_{k}^{l}$ valid for all $\mathbf{T}, R_{0}$ and $\mathbf{S}$. Explanations of how $T C_{k}^{l}$ $\forall k$ can be obtained are given in Section 4.2. The second step is to define $\overline{T C}$ as the lowest total cost under all currently known policies, and use a heuristic to determine a good initial value for $\overline{T C}$ (a close to optimal initial solution will provide tighter bounds). The heuristic solution is determined by first fixing the shipment intervals to some near optimal values $\overline{\mathbf{T}}$, i.e. $T_{k}=\bar{T}_{k} \forall k$, and then optimizing $R_{0}$ and $\mathbf{S}$. Values for $\bar{T}_{k} \forall k$ can be obtained from the deterministic Economic Order Interval (EOI) heuristic, presented in Marklund (2011). It does not allow for shortages and assumes that the demand per time unit at retailer $i$ is constant and equal to $\lambda_{i} \mu_{i}$. Noting that the shipment interval affects the inventory both at the warehouse and at the retailers, the expected cost per time unit for retailer group $k$ can be expressed as $\sum_{i \in N_{k}}\left(h_{0}+h_{i}\right) \bar{T}_{k} \frac{\lambda_{i} \mu_{i}}{2}+\frac{\omega_{k}}{\bar{T}_{k}}$. Minimizing this cost with respect to $\bar{T}_{k}$ renders

$$
\bar{T}_{k}=\sqrt{\frac{2 \omega_{k}}{\sum_{i \in N_{k}}\left(h_{0}+h_{i}\right) \lambda_{i} \mu_{i}}} .
$$


Given the shipment intervals $\bar{T}_{k} \forall k, R_{0}$ and $\mathbf{S}$ are optimized by searching the possible values of $R_{0}$ starting from $R_{0}^{l}=-Q_{0}$ (this bound is known from previous research, see for example Marklund 2011 and Axsäter 1998), and using the convexity property in Proposition 3 to optimize $S_{i} \forall i$ for each value of $R_{0}$. The search is limited by the upper bound $R_{0}^{u}$ in Proposition 4 . Note that $\overline{T C}$ and thereby $R_{0}$ are updated during the search as better solutions with lower expected costs are found.

Proposition 4. An upper bound for the optimal reorder point at the central warehouse, $R_{0}^{u}$, is obtained for the smallest value of $R_{0}$ satisfying the condition

$$
h_{0} E\left[I L_{0}^{+}\right] \geq \overline{T C}-\sum_{k \in K} T C_{k}^{l}
$$

For systems with fill rate constraints at the retailers, the optimization of $R_{0}$ and $\mathbf{S}$ (given $\mathbf{T}$ ) is achieved by searching the interval $\left[R_{0}^{l}, R_{0}^{u}\right]$, and for each $R_{0}$ determine the smallest $S_{i} \forall i$ satisfying the fill rate constraint at retailer $i$, utilizing that the expected holding costs are increasing in $S_{i}$.

The third step in the optimization procedure is to determine lower and upper bounds for the optimal $T_{k} \forall k \in K$, using Proposition 5 .

Proposition 5. For the optimal shipment interval of retailer group $k$, a lower bound is obtained by

$$
T_{k}^{l}=\frac{\overline{T C}-\sum_{\kappa \neq k} T C_{\kappa}^{l}-\sqrt{\left(\overline{T C}-\sum_{\kappa \neq k} T C_{\kappa}^{l}\right)^{2}-2 \omega_{k} \sum_{i \in N_{k}} h_{0} \lambda_{i} \mu_{i}}}{\sum_{i \in N_{k}} h_{0} \lambda_{i} \mu_{i}},
$$

and an upper bound by

$$
T_{k}^{u}=\frac{\overline{T C}-\sum_{\kappa \neq k} T C_{\kappa}^{l}+\sqrt{\left(\overline{T C}-\sum_{\kappa \neq k} T C_{\kappa}^{l}\right)^{2}-2 \omega_{k} \sum_{i \in N_{k}} h_{0} \lambda_{i} \mu_{i}}}{\sum_{i \in N_{k}} h_{0} \lambda_{i} \mu_{i}} .
$$

Finally, the optimal solution with respect to $\mathbf{T}, R_{0}$ and $\mathbf{S}$ is obtained by searching all combinations of $T_{k}$ within the bounded region, (using some step size $\tau$ ) and optimizing $R_{0}$ and $\mathbf{S}$ for every combination according to the same procedure as for the initial solution (where $T_{k}=\bar{T}_{k} \forall k$ ) explained above. As better solutions with lower total expected costs are found, $\overline{T C}$ is updated and the bounds on the shipment intervals and $R_{0}$ are tightened. When the search is complete, the optimal solution is found (the optimal system parameters for $T_{k} \forall k, R_{0}$ and $S_{i} \forall i$ are denoted $T_{k}^{*}, R_{0}^{*}$ and $S_{i}^{*}$ respectively) and the associated minimum expected cost, $T C^{*}$, equals $\overline{T C}$. Note that the probability mass functions of the warehouse backorders, which can be time consuming to calculate, are independent of $T_{k}$ and $\mathbf{S}$ and only need to be determined once for each $R_{0} \in\left[R_{0}^{l}, R_{0}^{u}\right]$. Moreover, as $\overline{T C}$ is updated during the optimization $R_{0}^{u}$ never increases. Thus, the probability mass functions of the warehouse backorders 
for the initial solution is sufficient for the entire optimization.

\subsection{Lower Bound for Costs Directly Related to Retailer Group $k$}

The presented bounds on $T_{k}$ and $R_{0}$ are based on the existence of a lower bound for all costs directly related to retailer group $k, T C_{k}^{l}$, valid for all values of $R_{0}$ and $T_{k}$. Lemma 4 provides such a bound which is applicable both for systems with backorder costs and fill rate constraints.

\section{Lemma 4.}

$$
T C_{k}^{l}=\sqrt{2 h_{0} \omega_{k} \sum_{i \in N_{k}} \lambda_{i} \mu_{i}} .
$$

The optimization of the retailer order-up-to levels is more time consuming for systems with backorder costs than for systems with fill rate constraints, as a numerical integration is needed when evaluating the expected retailer costs for each value of $S_{i}$. To compensate for this, Lemma 5 provides tighter bounds for $T C_{k}^{l}$ in backorder cost systems.

Lemma 5. A lower bound for the costs directly related to retailer group $k, T C_{k}\left(R_{0}, S, T_{k}\right)$ for all $R_{0}$, can for systems with backorder costs be obtained by minimizing these costs with respect to $T_{k}$ and $S_{i}$ for a system where there are no backorders at the central warehouse $\left(R_{0} \rightarrow \infty\right)$ :

$$
T C_{k}^{l}=\min _{T_{k}, S_{i} \forall i \in N_{k}}\left[T C_{k} \mid B_{i}\left(t_{0}\right)=0, \forall i \in N_{k}\right] \leq T C_{k}\left(R_{0}, S, T_{k}\right), \forall R_{0}, S, T_{k}
$$

$T C_{k}^{l}=\min _{T_{k}, S_{i}}\left(T C_{k} \mid B_{i}\left(t_{0}\right)=0, \forall i\right)$, may be computed by the algorithm in the online Appendix A.

\section{$5 \quad$ Numerical Results}

This section summarizes some of the results from a numerical study detailed in Appendix D found in the electronic companion to this paper. The purposes of this study are to investigate: the behavior of the optimal solutions, investigate the quality of the optimality bounds, and the performance of the EOI heuristic for determining the shipment intervals. This section focuses on results concerning the latter two. It also includes a discussion about computational issues. The study covers 128 problems representing all combinations of a high and a low value for the parameters $N, L_{i}, \rho_{i}$ (variance to mean ratio of the demand per time unit), $\beta_{i}, \omega_{k}, L_{0}$ and $Q_{0}$. More precisely, the number of retailers, $N=3$ or 6 ; where in both cases we have two retailer groups. When $N=3$, retailers $\{1,2\}$ constitute one group and retailer 3 a second. When $N=6$, retailers $\{1,2,3,4\}$ constitute one group and retailers $\{5$, $6\}$ a second. The expected demand per time unit, $E\left[D_{i}\right]$, is $\{2,1,3\}$ when $N=3$, and $\{2,1,2,1,4,2\}$ 
when $N=6$. The shipment times, $L_{i}=\{1,2,1\}$ or $\{2,4,2\}$ when $N=3$, and $\{1,1,2,2,1,2\}$ or $\{2,2,4,4,2,4\}$ when $N=6$. We also consider $\rho_{i}=1$ (Poisson demand) or 5 (compound Poisson demand with logarithmic compounding distributions) $\forall i \in N, \beta_{i}=10$ or $100 \forall i \in N, \omega_{k}=10$ or $100 \forall k \in K, L_{0}=1$ or $5, Q_{0}=2$ or 20 , and $h_{i}=1 \forall i$.

For every problem, $T_{k} \forall k \in K, R_{0}$ and $S_{i} \forall i \in N$ are optimized using the method in Section 4 (with step size $\tau=0.01$ time units for $T_{k} \forall k$ ). For the optimal solution we compute the distance to the optimality bounds, $\Delta R_{0}^{u}=R_{0}^{u}-R_{0}^{*}, \Delta T_{k}^{l}=T_{k}^{*}-T_{k}^{l}$ and $\Delta T_{k}^{u}=T_{k}^{u}-T_{k}^{*}$. We also determine the relative difference between optimal shipment intervals and those obtained by the EOI heuristic, $\Delta T_{k}=\left(\bar{T}_{k}-T_{k}^{*}\right) / T_{k}^{*}$, and the relative increase in the associated costs, $\Delta C=\left(\overline{T C}-T C^{*}\right) / T C^{*}$. The average, maximum and minimum results across all problems are presented in Table 1. From there we can see that the upper bound, $R_{0}^{u}$, which is the most important bound computationally, is rather tight; on average 7.41 units above $R_{0}^{*}$. The importance of $R_{0}^{u}$ stems from the fact that the warehouse backorder distributions are the most time consuming to compute (especially when $R_{0}+Q_{0}$ is large). Fortunately, they only need to be computed once for each $R_{0}$.

Table 1: Summary results for the numerical study

\begin{tabular}{c|ccccccccc}
\hline \hline & $E\left[T C^{*}\right]$ & $E\left[R_{0}^{*}\right]$ & $E\left[S_{i}^{*}\right]$ & $E\left[T_{k}^{*}\right]$ & $E[\Delta C]$ & $E[\Delta T]$ & $E\left[\Delta R_{0}^{u}\right]$ & $E\left[\Delta T_{k}^{l}\right]$ & $E\left[\Delta T_{k}^{u}\right]$ \\
\hline \hline Average & 111.51 & 19.89 & 19.41 & 3.15 & $0.14 \%$ & $2.99 \%$ & 7.41 & 2.19 & 23.55 \\
\hline \hline Minimum & 31.61 & -10 & 7.67 & 1.09 & $0.00 \%$ & $-9.79 \%$ & 0 & 0.91 & 7.05 \\
Maximum & 264.19 & 68 & 38.33 & 6.11 & $0.66 \%$ & $18.60 \%$ & 24 & 4.23 & 51.26 \\
\hline
\end{tabular}

Looking at the bounds for $T_{k}$, Table 1 shows that $T_{k}^{l}$ is on average 2.19 time units below the optimal value $T_{k}^{*}$, while $T_{k}^{u}$ exceeds it with on average 23.55 time units. Thus, the lower bound tends to be rather close to the optimum, while the upper bound is looser. Fortunately, from a computational perspective it is less important to provide tight bounds on $T_{k}^{*}$ than on $R_{0}^{*}$.

Table 1 indicates that the EOI heuristic performs well and offers a viable option to the optimal solution. On average it overestimates the shipment intervals by $2.99 \%$, but the average cost increase is only $0.14 \%$ with a maximum of $0.66 \%$. This suggests that the total cost is insensitive to the choice of shipment intervals around the optimum as long as $R_{0}$ and $\mathbf{S}$ are adjusted accordingly.

The computational complexity of the presented method is directly related to the number of combinations that need to be considered in the cost and fill rate evaluations (follows from the analysis in Section 3 and 4). Consequently, evaluation and optimization of large systems, with many retailers, high demand items, and long lead-times can be time consuming. Looking at the computation times for the problems in the numerical study they range from seconds to several hours, with changes in $L_{0}$ and $N$ having the biggest impact. On average, the computation times are 19 times longer for the problems with $L_{0}=5$ than for $L_{0}=1$, and 6.5 times longer for problems with $N=6$ (with $E\left[D_{0}\right]=12$ ) than 
for $N=3$ (with $E\left[D_{0}\right]=6$ ). In contrast, 10 fold increases of $\beta_{i}, \omega_{k}$ and $Q_{0}$ increase the computation times on average by $26 \%, 7 \%$, and $30 \%$ respectively. To investigate the computational impact of larger expected warehouse demand for fixed $N$, we solved 64 additional problems with $N=3$ for which the expected demand per time unit at each retailer was doubled rendering $E\left[D_{0}\right]=12$ (keeping the compounding distributions and everything else fixed). On average the computation times then increase by a factor 2.2. Thus, the computational times increase significantly with both $N$ and $E\left[D_{0}\right]$. Increasing the number of retailer groups $K$ (for fixed $N$ ) increases the number of candidate solutions to search in the optimization, but it only affects the inventory cost and fill rate calculations if the bounds $T_{k}^{l}$ and $T_{k}^{u}$ change so new $T_{i}$ - values need to be considered. For given optimality bounds and step sizes $\tau_{k}$, there are $\left(R_{0}^{u}-R_{0}^{l}\right) \prod_{k=1}^{K}\left(\left(T_{k}^{u}-T_{k}^{l}\right) / \tau_{k}\right)$ combinations of $R_{0}$ and $\mathbf{T}$ to consider. This means that the search space (and thereby the computation times) increases rapidly with $K$ if $\tau_{k}$ is small compared to $\left(T_{k}^{u}-T_{k}^{l}\right)$ for all $k$, but more modestly otherwise.

\section{$6 \quad$ Multi-Item Systems}

This section extends the analysis to multi-item systems, where $J$ items are controlled simultaneously. The items are distributed via a central warehouse to $N$ retailers (The model is also applicable to situations where different items are located at different central warehouses and shipments from these warehouses are consolidated). The items may be distributed in any way across the retailers. However, without loss of generality, we assume that each item is sold by at least one of the retailers and each retailer sells at least one of the items. Shipments may be consolidated to $K$ consolidation groups across items and retailers. A consolidation group consists of a number of items jointly distributed to a collection of retailers. In principle there can be different consolidation groups associated with different items, but $K \leq N \times J$. Note that in the single-item case a consolidation group is equivalent to a retailer group. From a practical perspective, the possibility to consolidate shipments across items as well as retailers increases both the practical applicability and the possible gain. We define:

$R_{0, j}$ : reorder point at the central warehouse for item $j S_{i, j}$ : order-up-to level at retailer $i$ for item $j$

$\mathbf{S}_{j}$ : vector with order-up-to levels for all retailers for item $j\left(S_{1, j}, \ldots, S_{N, j}\right)$

$T_{i, j}$ : shipment interval for item $j$ to retailer $i\left(=T_{k}\right.$ for all items and retailers in group $\left.k\right)$

$\mathbf{T}$ : matrix with shipment intervals for all items to all retailers $\left(T_{1,1}, \ldots, T_{1, J} ; \ldots ; T_{N, 1}, \ldots, T_{N, J}\right)$

$H C_{0, j}\left(R_{0, j}, \mathbf{T}\right)$ : holding costs at central warehouse for item $j$

$H C_{i, j}\left(R_{0, j}, \mathbf{S}_{j}, \mathbf{T}\right)$ : holding costs at retailer $i$ for item $j$

$B C_{i, j}\left(R_{0, j}, \mathbf{S}_{j}, \mathbf{T}\right)$ : backorder costs at retailer $i$ for item $j$ 
$T C_{M}:$ the total cost function in the multi-item system

As explained in Section 2, the fixed shipment costs are incurred for every scheduled shipment that leave the warehouse. In the multi-item case, the fixed cost for every scheduled shipment to consolidation group $k, \omega_{k}$, may, for example, be determined as the sum of the fixed shipment costs per retailer and item belonging to this group. This way to model the costs will assure that consolidation can be obtained both across retailers and items in group $k$ by using the same shipment intervals, and by synchronizing their start. In the cost functions for multi-item systems, the total shipment costs per time unit can still be determined as $S C(\mathbf{T})=\sum_{k \in K} \frac{\omega_{k}}{T_{k}}$.

Given $\mathbf{T}$, the holding and backorder costs as well as the fill rates for item $j$ are independent of the other items and can be determined as in the single-item case. The total cost function for the multi-item system with backorder costs is

$$
T C_{M}=\sum_{j \in J}\left[H C_{0, j}\left(R_{0, j} \mathbf{T}\right)+\sum_{i \in N}\left[H C_{i, j}\left(R_{0, j}, \mathbf{S}_{j}, \mathbf{T}\right)+B C_{i, j}\left(R_{0, j}, \mathbf{S}_{j}, \mathbf{T}\right)\right]+S C(\mathbf{T})\right.
$$

For a multi-item system with fill rate constraints, each item $j$ at retailer $i$ may have an individual fill rate constraint while the backorder costs are excluded from the total cost function. The fill rate of item $j$ at retailer $i, \gamma_{i, j}$, can be determined analogously to the single-item case using (10).

The optimization of multi-item systems is similar to the single-item optimization but with the coupling constraint that all items in a consolidation group use the same shipment interval. The shipment intervals are the only way in which the different items affect each other. The bounds for the warehouse reorder points need to be determined separately for each item, while $T_{k}^{l}$ and $T_{k}^{u}$ are only determined once for each consolidation group, taking all items into consideration. Propositions 4 and 5 can still be used to determine these bounds, provided that $\overline{T C}$ is redefined as the lowest total cost for all items under all currently known policies, and $T C_{k}^{l}$ is redefined as a lower bound for the total costs of all items related to consolidation group $k . T C_{k}^{l}$ can be obtained from Lemma 4 by including the holding costs for the reserved stock on hand of all items in group $k$.

The optimization procedure is analogous to the single-item case. First, $T C_{k}^{l}$ is determined. Second, an initial total cost, $\overline{T C}$, is obtained by optimizing a system where the shipment intervals, $\bar{T}_{k} \forall k \in$ $K$, are determined by a straightforward extension of the heuristic in (29). In this extension, the total holding cost is a summation of the holding costs of all items associated with the considered consolidation group. The optimization, given $\bar{T}_{k}$, is performed separately for each item $j \in J$ according to the single-item procedure. Given $\overline{T C}$, initial values for $T_{k}^{l}$ and $T_{k}^{u} \forall k \in K$ can be determined and 
the bounds are tightened as $\overline{T C}$ is updated. Finally, an optimal solution is found by searching all combinations of shipment intervals within the bounds, optimizing the reorder points and order-up-to levels at all retailers for all items separately, and choosing the solution with the lowest total cost.

\section{Summary, Extensions and Future Research}

In this paper we have presented a method for exact analysis of the inventory level distributions, fill rates and expected costs in one warehouse multi-retailer inventory systems with time based shipment consolidation and compound Poisson demand. An optimization procedure is also provided based on bounding the optimal decision variables; the warehouse reorder point, the retailer base-stock levels, and the shipment intervals. The method is applicable for both single- and multi-item systems.

The analysis can be extended to other related systems, for example with different replenishment policies at the central warehouse. The same technique for analyzing the inventory levels, costs and fill rates at the retailers (via the backorder distribution at the warehouse) can be used for any warehouse replenishment policy for which the inventory position and lead time distributions are known. One such extension is the use of an $(s, S)$ policy at the warehouse. For this system, the lead time $\left(L_{0}\right)$ is constant and the distribution of the warehouse inventory position can for instance be obtained by the technique in Axsäter (2006, p. 107-109). With this distribution known, the cost analysis of the entire system can be obtained analogously to the approach presented in this paper. Assuming that $S-s$ is fixed, the optimization can be handled analogously as well. Relaxing this assumption offers interesting challenges for further research.

Another possible extension is to let the warehouse use, what we refer to as, synchronized $(R, n Q)$ replenishments. This implies that replenishment orders from the central warehouse are placed only when there is a shipment leaving (to any retailer group) exactly $L_{0}$ time units later. To elaborate, consider a situation when the inventory position at the warehouse falls below $R_{0}$ at time $t_{0}$ and the first shipment from the warehouse after time $t_{0}+L_{0}$ leaves at $t_{0}+L_{0}+\Delta$. If the replenishment order of $Q_{0}$ units is placed at time $t_{0}$ (as our policy prescribes) it will arrive $\Delta$ time units before it is needed. Under the synchronized policy the warehouse delays the order placement $\Delta$ time units, and will thereby reduce its inventory with $Q_{0}$ units for $\Delta$ time units. Because of the periodic shipment schedule, this delay does not affect the service to the retailers. The analysis of the retailers in this system therefore remains the same as in our current model. However, the analyses of the warehouse inventory (both the reserved and unreserved stock on hand) and the optimization bounds are severely complicated and beyond the scope of this paper to solve. Note that, theoretically, the synchronized policy offers a performance guarantee over the presented $(R, n Q)$ policy. Intuitively, the difference in 
performance will depend on how often shipments are dispatched. This is influenced, for example, by the number of retailer/consolidation groups, the length of the shipment intervals and the coordination of dispatching times. Frequent shipments suggests smaller opportunities for savings. From a practical perspective, there are two drawbacks with the synchronized policy; (1) it is sensitive to variations in the replenishment lead times, $L_{0}$, and (2) the receiving and loading activities of all items at the central warehouse are concentrated to the same time instances. Other possible extensions would be periodic $(R, n Q)$ replenishments, and synchronized or periodic $(s, S)$ policies.

The exact approach presented in this paper can be computationally challenging to apply to large systems. Thus, another direction for future research is to consider computationally more efficient methods for cost analysis and optimization. In addition, we believe that our analysis provides a good foundation for future research on both exact methods and accurate heuristics for analyzing similar divergent systems. Other possible future areas of research using extensions of this approach includes $N$-echelon distribution systems, periodic replenishments to the central warehouse or demand with other distributions than compound Poisson.

\section{Electronic Companion}

An electronic companion, containing all proofs and appendices, is available together with the online version of this paper at http://or.journal.informs.org/.

\section{References}

Andersson, J., S. Axsäter, J. Marklund. 1998. Decentralized multiechelon inventory control. Production and Operations Management 7 370-386.

Andersson, J., J. Marklund. 2000. Decentralized inventory control in a two-level distribution system. European Journal of Operational Research 127 483-506.

Axsäter, S. 1990. Simple Solution Procedures for a Class of Two-Echelon Inventory Problems. Operations Research 38 $64-69$.

Axsäter, S. 1993a. Exact and approximate evaluation of batch-ordering policies for two-level inventory systems. Operations Research 41 777-785.

Axsäter, S. 1993b. Optimization of order-up-to- $S$ policies in two-echelon inventory systems with periodic review. Naval Research Logistics $\mathbf{4 0} 245-253$.

Axsäter, S. 1996. Using the deterministic EOQ formula in stochastic inventory control. Management Science $42830-834$.

Axsäter, S. 1997. Simple evaluation of echelon stock $(R, Q)$-policies for two-level inventory systems. IIE Trans. 29 661-669.

Axsäter, S. 1998. Evaluation of installation stock based $(R, Q)$-policies for two-level inventory systems with Poisson demand. Operations Research 46 135-145.

Axsäter, S. 2000. Exact analysis of continuous review $(R, Q)$-policies in two-echelon inventory systems with compound Poisson demand. Operations Research 48 686-696. 
Axsäter, S. 2001. A note on stock replenishment and shipment scheduling for vendor-managed inventory systems. Management Science 44 1306-1310.

Axsäter, S. 2003. Supply chain operations: Serial and distribution inventory systems. Handbooks in operations research and management science. Vol.11: Supply chain management: Design, coordination and operation, S.C. Graves and A.G. de Kok (Editors), Elsevier, Amsterdam, The Netherlands, 525-559.

Axsäter S. 2006. Inventory Control. Springer: Berlin.

Axsäter S., J. Marklund, E.A. Silver. 2002. Heuristic Methods for Centralized Control of One-warehouse, N-Retailer Inventory Systems. Manufacturing and Service Operations Management 4 75-97.

Axsäter S., J. Marklund. 2008. Optimal "Position Based" Warehouse Ordering in Divergent Two-Echelon Inventory Systems. Operations Research 56 976-991.

Berling, P., J. Marklund. 2013. A model for heuristic coordination of real life distribution inventory systems with lumpy demand. European Journal of Operational Research 230 515-526.

Berling, P., J. Marklund. 2014. Multi-echelon inventory control: A normal demand model for implementation in practice. International Journal of Production Research 52 3331-3347.

Cachon G.P. 1999. Exact Evaluation of Batch-ordering Inventory Policies in Two-echelon Supply Chains with Periodic Review. Operations Research 49 79-98.

Cachon, G., M. Fisher. 2000. Supply chain inventory management and the value of shared information. Management Science 46 1032-1048.

Çetinkaya S., C.Y. Lee. 2000. Optimal outbound dispatch policies: Modeling inventory and cargo capacity. Management Science 46 217-232.

Çetinkaya, S., J.H. Bookbinder. 2003. Stochastic Models for the Dispatch of Consolidated Shipments. Transportation Research 37 747-768.

Çetinkaya, S., E. Tekin, C.Y. Lee. 2008. A Stochastic Model for Joint Inventory and Outbound Shipment Decisions. IIE Transactions 40 324-340.

Chen, F., Y.S. Zheng. 1997. One Warehouse Multi-Retailer Systems with Centralized Stock Information. Operations Research 45 275-287.

Cheung K.L., H.L. Lee. 2002. The Inventory Benefit of Shipment Coordination and Stock Rebalancing in a Supply Chain. Management Science 48 300-306.

Chu L.Y., Z.M. Shen. 2010. A Power-of-Two Ordering Policy for One-Warehouse Multi-retailer Systems with Stochastic Demand. Operations Research 58 492-502.

Diks, E.B., A.G. de Kok. 1998. Optimal control of a divergent multi-echelon inventory system. European Journal of Operational Research 111 75-97.

Diks, E.B., A.G. de Kok. 1999. Computational results for the control of a divergent $N$-echelon inventory system. International Journal of Production Economics 59 327-336.

Eaves A.H.C. 2002. Forecasting for the ordering and stock- holding of consumable spare parts. PhD Thesis, University of Lancaster.

Federgruen, A., P. Zipkin. 1984. Approximations of Dynamic Multilocation Production and Inventory Problems. Management Science 30 69-84.

Federgruen, A. 1993. Centralized Planning Models for Multi-Echelon Inventory Systems under Uncertainty, in S.C. Graves et al. Eds., Handbooks in OR $\mathcal{E}$ MS 4, North Holland Amsterdam, pp. 133-173.

Feller, W. 1968. An Introduction to Probability Theory and its Applications, Vol. 1, 3rd edition, John Wiley \& Sons, New York.

Forsberg, R. 1995. Optimization of order-up-to- $S$ policies for two-level inventory systems with compound Poisson demand. European Journal of Operational Research 81 143-153.

Forsberg, R. 1997. Exact evaluation of $(R, Q)$-policies for two-level inventory systems with Poisson demand. European Journal of Operational Research 96 130-138.

Gaur, V., M. L. Fisher, 2004. A periodic inventory routing problem at a supermarket chain. Operations Research 32 1019-1037.

Graves, S.C. 1985. A Multi-Echelon Inventory Model for a Repairable Item with One-for-One Replenishment. Management Science 31 1247-1256. 
Graves, S.C. 1996. A multi-echelon inventory model with fixed replenishment intervals. Management Science 42 1-18.

Gürbüz, M., K. Moinzadeh, Y.P. Zhou. 2007. Coordinated replenishment strategies in inventory/distribution systems. Management Science 53 293-307.

Heijden M.C. van der, E.B. Diks, A.G. de Kok, 1997. Stock Allocation in General Multi-Echelon Distribution Systems with (R, S) Order-up-to-policies, International Journal of Production Economics 49 157-174.

Houtum G.J. van, K. Inderfurth, W.H.M. Zijm. 1996. Materials Coordination in Stochastic Multi-Echelon Systems. European Journal of Operational Research 95 1-23.

Howard, C. 2013. New Allocation Policies for Divergent Inventory Systems with Real-time Information and Shipment Consolidation, in Howard, C., Real-time Allocation Decisions in Multi-echelon Inventory Control, Doctoral Thesis, Div. of Production Management, Dept. of Industrial Management and Logistics, Lund University, Faculty of Engineering, 2013, ISBN 978-91-7473-509-3, Media-Tryck, Lund University, Lund.

Howard C., J. Marklund. 2011. Evaluation of stock allocation policies in a divergent system with shipment consolidation. European Journal of Operational Research 211 289-309.

Howard, C., O. Stenius 2013. Partial or Complete Deliveries in Two-echelon Inventory Systems?, in Howard, C., Realtime Allocation Decisions in Multi-echelon Inventory Control, Doctoral Thesis, Div. of Production Management, Dept. of Industrial Management and Logistics, Lund University, Faculty of Engineering, 2013, ISBN 978-91-7473-509-3, MediaTryck, Lund University, Lund.

Jackson, P.L. 1988. Stock Allocation in a Two-Echelon Distribution System or What to do Until Your Ship Comes In. Management Science 34 880-895.

Kapuscinski, R., Zhang, R.Q., Carbonneau, P., Moore, R. Reeves, B., 2004. Inventory Decisions in Dell's Supply Chain. Interfaces, 34, 191-205.

Kiesmüller, G.P., A.G. de Kok, S.R. Smits, P.J.M. van Laarhoven. 2004. Evaluation of divergent N-echelon (s,nQ)policies under compound renewal demand. OR Spectrum 26 547-577.

Kuhn, H., M.G. Sternbeck, 2013. Integrative retail logistics: An exploratory study. Operations Management Research 6 $2-18$.

Lengu D., A.A. Syntetos, , M.Z. Babai. 2014. Spare parts management: Linking distributional assumptions to demand classification. European Journal of Operational Research, 235, 624-635.

Marklund J. 2002. Centralized Inventory Control in a Two-Level Distribution System with Poisson Demand. Naval Research Logistics 49 798-822.

Marklund J. 2006. Controlling Inventories in Divergent Supply Chains with Advance-Order Information. Operations Research 54 988-1010.

Marklund, J. 2011. Inventory Control in Divergent Supply Chains with Time-Based Dispatching and Shipment Consolidation. Naval Research Logistics 58 59-71.

Marklund, J., K. Rosling. 2012. Lower bounds and heuristics for supply chain stock allocation, Operations Research 60 92-105.

Marquès, G., C. Thierry, J. Lamothe, D. Gourc. 2010. A review of Vendor Managed Inventory (VMI): from concept to processes. Production Planning and Control 21 547-561.

Moinzadeh K. 2002. A Multi-Echelon Inventory System with Information Exchange, Management Science 48 $414-426$.

Mutlu, F., S. Çetinkaya, J.H. Bookbinder, 2010. An analytical model for computing the optimal time-and-quantity based policy for consolidated shipments. IIE Transactions 42 367-377.

Özer, Ö. 2003. Replenishment strategies for distribution systems under advanced demand information. Management Science $49255-272$.

Shang, K., Z. Tao, S. Zhou. 2015. Optimizing reorder intervals for two-echelon distribution systems with stochastic demand. Operations Research 63 458-475.

Sherbrooke, C.C. 1968. METRIC: A Multi-Echelon Technique for Recoverable Item Control. Operations Research 16 $122-141$.

Sherbrooke, C.C. 1986. Vari-Metric: Improved Approximations for Multi-Indenture, Multi-Echelon Availability Models, Operations Research 34 311-319.

Simon, R.M. 1971. Stationary Properties of a Two Echelon Inventory Model for Low Demand Items. Operations Research $19761-777$. 
Zheng, Y.S. 1992. On properties of stochastic inventory systems. Management Science $\mathbf{3 8} 87-103$.

Zipkin P. 2005. Foundations of Inventory Management. McGraw-Hill Primis.

\section{Author Biography}

Olof Stenius is a PhD student at the department of Production Management at Lund University, Sweden. He holds a MSc in Industrial Engineering and Management, from Lund University, Faculty of Engineering. His research interests include inventory control, supply chain management, yield management and environmental management.

Ayşe Gönül Karaarslan is Assistant Professor of Econometrics at Erasmus University Rotterdam. She holds a PhD in Industrial Engineering from Eindhoven University of Technology and a MSc in Industrial Engineering from Bogaziçi University in İstanbul. Her research interests include supply chain management, reverse logistics, and machine learning.

Johan Marklund is Professor of Production Management at Lund University, Faculty of Engineering in Sweden. Previously he has held positions at the Leeds School of Business, University of Colorado, Boulder, and the Boston Consulting Group. He holds degrees from Linköping University (MSc), and Lund University (BSc and PhD). His research interests include inventory theory, supply chain management and logistics, with a special focus on stochastic multi-echelon inventory control problems.

A. G. de Kok is Professor of Quantitative Analysis of Logistics Systems at Eindhoven University of Technology, School of Industrial Engineering, in The Netherlands. Previously he held positions at Philips Electronics as senior consultant in Operations Research and Logistics Innovation Manager. He holds a PhD in Mathematics from the Free University in Amsterdam. From Leiden University he holds a MSc in Mathematics and a BSc in Mathematics and Physics. His research interests include supply chain management, hierarchical planning and concurrent engineering. 
E-Companion - Exact Analysis of Divergent Inventory Systems with Time-Based Shipment Consolidation and Compound Poisson Demand

Olof Stenius

Ayşe Gönül Karaarslan

Johan Marklund

A. G. de Kok 


\section{Appendix A: Algorithm for Determining $T C_{k}^{l}$}

To find the lower bound for the total costs directly associated with retailer group $k, T C_{k}$, in Lemma 5 ,

$$
T C_{k}^{l}=\min _{T_{k}, S_{i}}\left(T C_{k} \mid B_{i}\left(t_{0}\right)=0, \forall i\right),
$$

we go through the following steps:

1. Determine a near optimal shipment interval for retailer group $k, \bar{T}_{k}$, using (29)

2. Given $\bar{T}_{k}$, determine near optimal reorder points for each retailer $\bar{S}_{i}$. This is done by optimizing each retailer separately as a single-echelon system. Note that the convexity property in Proposition 3 holds also for systems where $B_{i}=0\left(R_{0} \rightarrow \infty\right)$.

3. Calculate $\overline{T C}_{k}=T C_{k}\left(\bar{T}_{k},{\overline{S_{i}}}\right)$.

4. Obtain upper and lower bounds for $T_{k}$ for the optimization. These bounds are obtained analogously to the bounds in Proposition 5 as

$$
\begin{aligned}
T_{k}^{l} & =\frac{\overline{T C}_{k}-\sqrt{\overline{T C_{k}^{2}-2 \omega_{k} \sum_{i \in N_{k}} h_{0} \lambda_{i} \mu_{i}}}}{\sum_{i \in N_{k}} h_{0} \lambda_{i} \mu_{i}} \\
T_{k}^{u} & =\frac{\overline{T C}_{k}+\sqrt{\overline{T C_{k}^{2}-2 \omega_{k} \sum_{i \in N_{k}} h_{0} \lambda_{i} \mu_{i}}}}{\sum_{i \in N_{k}} h_{0} \lambda_{i} \mu_{i}} .
\end{aligned}
$$

5. Search through all values of $T_{k}$ in this interval and optimize $S_{i}$ for each $T_{k}$ to find the lower bound, $T C_{k}^{l}$.

\section{Appendix B: Proofs}

\section{Proof of Proposition 1}

Proof. The reserved stock on hand at the warehouse increase whenever an unreserved unit on hand is reserved or a backordered unit arrives to the central warehouse. Thus the process by which the reserved stock on hand accumulates depends on the customer demand process, and the warehouse replenishment process. For fixed policies these two processes are in the current system independent of the shipment process, i.e., the length of the shipment interval, and when shipments leave the central warehouse. Hence, the same holds for the accumulation process of the reserved stock on hand. Moreover, as all unsatisfied demand is backordered, and all units will be reserved stock on hand at the warehouse at some point in time, the average rate by which the reserved stock on hand for retailer $i$ accumulate is equal to the demand rate at retailer $i, \lambda_{i} \mu_{i}$. Consequently, if the previous shipment to retailer $i$ left at time $t$, the expected number of units on hand at the warehouse reserved by retailer $i$, at $t+\tau$, for any $\tau \in\left(0, T_{i}\right]$, is $\lambda_{i} \mu_{i} \tau$. This means that the expected amount of reserved stock on hand for retailer $i$ will increase linearly between two consecutive shipments from 0 to $\lambda_{i} \mu_{i} T_{i}$. Taking the average over time renders (4). 


\section{Proof of Lemma 1:}

Proof. Conditioning on at least $n$ retailer orders during $\left(t_{0}-L_{0}, t_{0}\right], \Phi_{0} \geq n$, and that $\Psi^{n-1}=x$, for $x>0$, there will be $u>0$ partial backorders for retailer $i$ caused by the $n^{\text {th }}$ retailer order if two conditions are fulfilled: (i) The $n^{t h}$ retailer order originates from retailer $i$. The probability for this is $\frac{\lambda_{i}}{\lambda_{0}}$. (ii) The quantity of this order is $x+u$. The probability for this is $P\left\{Y_{i}=x+u\right\}$. This renders for $u>0$,

$$
P\left\{\hat{B}_{i}^{n}\left(t_{0}\right)=u \mid \Phi_{0} \geq n \text { and } \Psi^{n-1}=x\right\}=\frac{\lambda_{i}}{\lambda_{0}} P\left\{Y_{i}=x+u\right\}, \forall x>0 .
$$

(14) follows from unconditioning with respect to $\Phi_{0}$ and $\Psi^{n-1}$. The latter by considering all possible positive values $x \in\left[1, S_{0}-n+1\right]$.

In order to have 0 partial backorders for retailer $i$, when the $n^{\text {th }}$ retailer order after $t_{0}-L_{0}$ brings the nominal inventory position to a non-positive value, the proof is analogous. In this case, however, there are two different scenarios; either the $n^{\text {th }}$ customer arrives from retailer $i$ and demands exactly $x$ units to move the nominal inventory position to 0 , or the $n^{\text {th }}$ customer arrives from another retailer and demands more than or equal to $x$ units.

\section{Proof of Lemma 2:}

Proof. The distribution of complete backorders depends on $n$, the retailer order that brings the nominal inventory position to a non-positive value. However, because of the memoryless property of the compound Poisson demand, the complete backorders are independent of which retailers these $n$ first orders originated from, and the sizes of these orders.

Given that there are $a$ orders to retailer $i$ after the $n^{\text {th }}$ retailer order, i.e., $\check{\Phi}_{i, n}=a$, it is clear that $P\left\{\check{B}_{i, n}\left(t_{0}\right)=v \mid \check{\Phi}_{i, n}=a\right\}=P\left\{Y_{i}^{a}=v\right\}$. (17) follows by taking the expectation over all possible outcomes of $\check{\Phi}_{i, n}$.

To arrive at (18) we note that given $m$ retailer orders that are completely backordered, the probability that $a$ of these originates with retailer $i$ is $P\left\{Z_{i}^{m}=a\right\}$. Moreover, the probability of $n+m$ retailer orders in $\left(t_{0}-L_{0}, t_{0}\right]$ given at least $n$ orders in $\left(t_{0}-L_{0}, t_{0}\right]$ is $P\left\{\Phi_{0}=n+m\right\} / P\left\{\Phi_{0} \geq n\right\}$. (18) follows as an expectation over all possible values of $m$.

\section{Proof of Proposition 2:}

Proof. By definition $P\left\{\hat{B}_{i}^{n}\left(t_{0}\right)=u\right\}$ is the probability that the $n^{\text {th }}$ retailer order brings $\Psi^{n}$ to a nonpositive value, causing $u$ partial backorders for retailer $i$. Also, by definition $P\left\{\check{B}_{i, n}\left(t_{0}\right)=r-u\right\}$ is the probability that there are $r-u$ complete backorders for retailer $i$ conditioned on that the $n^{\text {th }}$ retailer order brings $\Psi^{n}$ to a non-positive value. Taking the expectation over all possible values of $n$ and $u$ (noting that $u \leq r$ ) renders $(20)$.

For $r=0$ we also need to consider the probability that the inventory position never reaches zero during the replenishment lead time, $p_{0}$, rendering (21). 


\section{Proof of Lemma 3:}

Proof. In order for $\tilde{B}_{i}^{-m}\left(t_{0}\right)=u$ there are two possible scenarios; (a) The $m^{\text {th }}$ order before $\left(t_{0}-L_{0}\right)$ arrives from retailer $j \neq i$ and the size of this order is at least $-\Psi^{-(m-1)}=-x$ units $(x<0)$, and (b) the $m^{\text {th }}$ order originates with retailer $i$ and is for at least $-\Psi^{-m}=-x$ units. Starting with (a) the probability that the $m^{\text {th }}$ customer arrives from retailer $j \neq i$ and demands more than $-x$ units is $\left(\lambda_{i^{c}} / \lambda_{0}\right) P\left\{Y_{i^{c}} \geq-x\right\}$. In order for $\tilde{B}_{i}^{-m}\left(t_{0}\right)=u$ in this scenario, the $m-1$ next customer orders need to contain $u$ units to retailer $i$ and need to assure that the nominal inventory position is $x$, which can be expressed as the probability $P\left\{V_{i}^{m-1}=u\right.$ and $\left.\Psi^{-(m-1)}=x\right\}$. A summation over all possible values of $x\left(x \in\left[S_{0}+m-1,-1\right]\right)$ generates the first part of $(25)$.

The probability for scenario (b) is $\left(\lambda_{i} / \lambda_{0}\right) P\left\{Y_{i} \geq-x\right\}$. In this scenario, $-x$ units of the $m^{\text {th }}$ order will be backordered at $t_{0}$. Thus, in order for $\tilde{B}_{i}^{-m}\left(t_{0}\right)=u$, the next $m-1$ customers need to order $u-(-x)=u+x$ units to retailer $i$ and ensure that $\Psi^{-(m-1)}=x$, which can be expressed as the probability $P\left\{V_{i}^{m-1}=u+x\right.$ and $\left.\Psi^{-(m-1)}=x\right\}$. A summation over all possible values of $x$ renders the second part of (25).

In order for $V_{i}^{m-1}=u$ and $\Psi^{-(m-1)}=x$, the last $m-1$ customer orders before $t_{0}-L_{0}$ need to include $u$ units to retailer $i$ and $x-S_{0}-u$ units to all other retailers (recall $S_{0}<0$ and $x<0$ ). With $Z_{i}^{m-1}$ defined as in Section 3.3.1 and determined by (16) we get (26).

\section{Proof of Proposition 3:}

Proof. Neither the shipment costs, $\sum_{k=1}^{K} \frac{\omega_{k}}{T_{k}}$, nor the holding costs at the central warehouse are affected by $S_{i}$. Furthermore, the holding cost and backorder cost at retailer $i$ are unaffected by the order-up-to levels at other retailers. Thus, for fixed $R_{0}$ and $\mathbf{T}, T C\left(R_{0}, \mathbf{S}, \mathbf{T}\right)$ is separable in the retailer order-up-to levels. To assert the convexity in $S_{i}$ we define the holding and backorder costs at retailer $i$ as

$$
R C_{i}\left(R_{0}, S_{i}, T_{i}\right)=h_{i} E\left[I L_{i}^{+}(s)\right]+\beta_{i} E\left[I L_{i}^{-}(s)\right] .
$$

It is sufficient to show convexity for $R C_{i}\left(R_{0}, S_{i}, T_{i}\right)$ with respect to $S_{i}$ for each retailer $i$. We define the difference function $\Delta G(s)$ as follows:

$$
\Delta G(s)=R C_{i}\left(R_{0}, s+1, T_{i}\right)-R C_{i}\left(R_{0}, s, T_{i}\right)
$$

To prove convexity, we need to show that $\Delta G(s)-\Delta G(s-1) \geq 0$. First, by using (7), (8) and (9), we rewrite $R C_{i}\left(R_{0}, s, T_{i}\right)$ as:

$$
\begin{aligned}
R C_{i}\left(R_{0}, s, T_{i}\right)= & \left(h_{i}+\beta_{i}\right) E\left[I L_{i}^{+}(s)\right]-\beta_{i} E\left[I L_{i}(s)\right] \\
= & \left(h_{i}+\beta_{i}\right) \frac{1}{T_{i}} \sum_{j=1}^{s} \sum_{r=0}^{s-j} j P\left\{B_{i}\left(t_{0}\right)=r\right\} \int_{0}^{T_{i}} P\left\{D_{i}\left(L_{i}+x\right)=s-j-r\right\} d x \\
& -\beta_{i}\left(s-E\left[B_{i}\left(t_{0}\right)\right]-\lambda_{i} \mu_{i} L_{i}\right) .
\end{aligned}
$$

Note that the probability mass function and expectation of $B_{i}\left(t_{0}\right)$ does not depend on the order-up-to 
levels of the retailers. Next, we derive $R C_{i}\left(R_{0}, s+1, T_{i}\right)$ in terms of $R C_{i}\left(R_{0}, s, T_{i}\right)$ :

$$
\begin{aligned}
R C_{i}( & \left.R_{0}, s+1, T_{i}\right)= \\
& \left(h_{i}+\beta_{i}\right) \frac{1}{T_{i}} \sum_{j=1}^{s+1} \sum_{r=0}^{s+1-j} j P\left\{B_{i}\left(t_{0}\right)=r\right\} \int_{0}^{T_{i}} P\left\{D_{i}\left(L_{i}+x\right)=s+1-j-r\right\} d x \\
& -\beta_{i}\left(s+1-E\left[B_{i}\left(t_{0}\right)\right]-\lambda_{i} \mu_{i} L_{i}\right), \\
= & \left(h_{i}+\beta_{i}\right) \frac{1}{T_{i}} \sum_{z=0}^{s} \sum_{r=0}^{s-z}(z+1) P\left\{B_{i}\left(t_{0}\right)=r\right\} \int_{0}^{T_{i}} P\left\{D_{i}\left(L_{i}+x\right)=s-z-r\right\} d x \\
& -\beta_{i}\left(s+1-E\left[B_{i}\left(t_{0}\right)\right]-\lambda_{i} \mu_{i} L_{i}\right), \\
= & \left(h_{i}+\beta_{i}\right) \frac{1}{T_{i}} \sum_{z=0}^{s} \sum_{r=0}^{s-z} z P\left\{B_{i}\left(t_{0}\right)=r\right\} \int_{0}^{T_{i}} P\left\{D_{i}\left(L_{i}+x\right)=s-z-r\right\} d x \\
& +\left(h_{i}+\beta_{i}\right) \frac{1}{T_{i}} \sum_{z=0}^{s} \sum_{r=0}^{s-z} P\left\{B_{i}\left(t_{0}\right)=r\right\} \int_{0}^{T_{i}} P\left\{D_{i}\left(L_{i}+x\right)=s-z-r\right\} d x \\
& -\beta_{i}\left(s-E\left[B_{i}\left(t_{0}\right)\right]-\lambda_{i} \mu_{i} L_{i}\right)-\beta_{i}, \\
= & \left(h_{i}+\beta_{i}\right) \frac{1}{T_{i}} \sum_{z=0}^{s} \sum_{r=0}^{s-z} P\left\{B_{i}\left(t_{0}\right)=r\right\} \int_{0}^{T_{i}} P\left\{D_{i}\left(L_{i}+x\right)=s-z-r\right\} d x \\
& -\beta_{i}+R C_{i}\left(R_{0}, s, T_{i}\right) .
\end{aligned}
$$

As a result the first order difference is equal to:

$$
\Delta G(s)=\left(h_{i}+\beta_{i}\right) \frac{1}{T_{i}} \sum_{j=0}^{s} \sum_{r=0}^{s-j} P\left\{B_{i}\left(t_{0}\right)=r\right\} \int_{0}^{T_{i}} P\left\{D_{i}\left(L_{i}+x\right)=s-j-r\right\} d x-\beta_{i} .
$$

By following the similar line of thought, we get the following for the second order difference:

$$
\Delta G(s)-\Delta G(s-1)=\left(h_{i}+\beta_{i}\right) \frac{1}{T_{i}} \sum_{r=0}^{s} P\left\{B_{i}\left(t_{0}\right)=r\right\} \int_{0}^{T_{i}} P\left\{D_{i}\left(L_{i}+x\right)=s-r\right\} d x \geq 0 .
$$

\section{Proof of Proposition 4:}

Proof. It is clear from (3) that $h_{0} E\left[I L_{0}^{+}\right]$is increasing in $R_{0}$ for all values of $R_{0} \geq-Q_{0}$ and that the total cost $T C\left(R_{0}, \mathbf{S}, \mathbf{T}\right)=h_{0} E\left[I L_{0}^{+}\right]+\sum_{k \in K} T C_{k}\left(R_{0}, \mathbf{S}, T_{k}\right) \geq h_{0} E\left[I L_{0}^{+}\right]+\sum_{k \in K} T C_{k}^{l}$. With $R_{0}^{u}=\min \left\{R_{0}: h_{0} E\left[I L_{0}^{+}\right]+\sum_{k \in K} T C_{k}^{l} \geq \overline{T C}\right\}$ it follows that for all $R_{0}>R_{0}^{u}, T C\left(R_{0}, \mathbf{S}, \mathbf{T}\right) \geq \overline{T C}$ and searching this region cannot reduce the costs. 


\section{Proof of Proposition 5:}

Proof. Let

$$
\Theta_{k}\left(T_{k}\right)=h_{0} \sum_{i \in N_{k}} E\left[W_{i}\right]+\frac{\omega_{k}}{T_{k}}=\frac{1}{2} \sum_{i \in N_{k}} h_{0} \lambda_{i} \mu_{i} T_{k}+\frac{\omega_{k}}{T_{k}},
$$

be the costs directly related to shipment interval $T_{k}$. Note that $\Theta_{k}\left(T_{k}\right)$ is strictly convex in $T_{k}$ as (for $\left.T_{k}>0\right)$

$$
\frac{\partial^{2} \Theta_{k}}{\partial T_{k}^{2}}=\frac{\partial^{2}\left(\frac{1}{2} \sum_{i \in N_{k}} h_{0} \lambda_{i} \mu_{i} T_{k}+\frac{\omega_{k}}{T_{k}}\right)}{\partial T_{k}^{2}}=0+2 \frac{\omega_{k}}{T_{k}^{3}}>0 .
$$

Note also that

$$
\begin{aligned}
& T C\left(R_{0}, \mathbf{S}, \mathbf{T}\right)=h_{0} E\left[I L_{0}^{+}\right]+\sum_{\kappa \in K} T C_{\kappa}\left(R_{0}, \mathbf{S}, T_{\kappa}\right) \\
& \geq T C_{k}\left(R_{0}, \mathbf{S}, T_{k}\right)+\sum_{\kappa \in K \backslash\{k\}} T C_{\kappa}\left(R_{0}, \mathbf{S}, T_{\kappa}\right) \geq \Theta_{k}\left(T_{k}\right)+\sum_{\kappa \in K \backslash\{k\}} T C_{\kappa}^{l} .
\end{aligned}
$$

It follows that no $T_{k}$ satisfying

$$
\Theta_{k}\left(T_{k}\right) \geq \overline{T C}-\sum_{\kappa \in K \backslash\{k\}} T C_{\kappa}^{l}
$$

can render a lower total expected cost than $\overline{T C}$. From (B8), (B11) and the convexity of $\Theta_{k}\left(T_{k}\right)$ we arrive at (31) and (32) by solving

$$
\frac{1}{2} \sum_{i \in N_{k}} h_{0} \lambda_{i} \mu_{i} T_{k}+\frac{\omega_{k}}{T_{k}}=\overline{T C}-\sum_{\kappa \in K \backslash\{k\}} T C_{\kappa}^{l}
$$

with respect to $T_{k}$. When $T_{k}$ is smaller than the smallest root, $T_{k}^{l}$, or larger than the largest root, $T_{k}^{u}$, (B11) is always satisfied and these regions do not need to be searched.

\section{Proof of Lemma 4:}

Proof. From Proposition 5 we know that the costs directly related to the shipment interval $T_{k}$, $\Theta_{k}\left(T_{k}\right)=\sum_{i \in N_{k}} E\left[W_{i}\right]+\frac{\omega_{k}}{T_{k}}$ is convex in $T_{k}$. Hence, the shipment interval $T_{k, l}$ that minimizes $\Theta_{k}\left(T_{k}\right)$ is obtained from the first order optimality condition

$$
\frac{\delta \Theta_{k}}{\delta T_{k}}=\frac{\delta}{\delta T_{k}}\left(\frac{1}{2} \sum_{i \in N_{k}} h_{0} \lambda_{i} \mu_{i} T_{k}+\frac{\omega_{k}}{T_{k}}\right)=0,
$$

which renders

$$
T_{k, l}=\sqrt{\frac{2 \omega_{k}}{\sum_{i \in N_{k}} h_{0} \lambda_{i} \mu_{i}}} .
$$

(33) follows from

$$
T C_{k}\left(T_{k}\right) \geq \Theta_{k}\left(T_{k}\right) \geq \Theta_{k}\left(T_{k, l}\right)=\sqrt{2 h_{0} \omega_{k} \sum_{i \in N_{k}} \lambda_{i} \mu_{i}}
$$

\section{Proof of Lemma 5:}

Proof. From (27) $T C_{k}\left(R_{0}, \mathbf{S}, T_{k}\right)=\frac{\omega_{k}}{T_{k}}+\sum_{i \in N_{k}} h_{0} E\left[W_{i}\right]+h_{i} E\left[I L_{i}^{+}\right]+\beta_{i} E\left[I L_{i}^{-}\right]$. Note that neither $W_{i}$ nor $\frac{\omega_{k}}{T_{k}}$ depend on $R_{0}$. Moreover, the retailer costs, $R C_{i}\left(R_{0}, S_{i}, T_{k}\right)=h_{i} E\left[I L_{i}^{+}(s)\right]+\beta_{i} E\left[I L_{i}^{-}(s)\right]$, 
depend on $R_{0}$ solely through the backorder distribution, $B_{i}\left(t_{0}\right)$. From (6) we can show that the probability of an inventory level $j$ at time $t_{0}+L_{i}+t$ at retailer $i$ when $B_{i}\left(t_{0}\right)=b_{i}$ is

$$
\begin{aligned}
P\left\{I L_{i}\left(t_{0}+L_{i}+t\right)=j\right\} & =P\left\{b_{i}+D_{i}\left(t_{0}, t_{0}+L_{i}+t\right)=S_{i}-j\right\} \\
& =P\left\{\left(b_{i}+1\right)+D_{i}\left(t_{0}, t_{0}+L_{i}+t\right)=\left(S_{i}+1\right)-j\right\},
\end{aligned}
$$

which implies that

$$
R C_{i}\left(S_{i}, T_{k} \mid B_{i}\left(t_{0}\right)=b_{i}\right)=R C_{i}\left(S_{i}+1, T_{k} \mid B_{i}\left(t_{0}\right)=b_{i}+1\right) .
$$

For reasons of exposition and without loss of generality we renumber the retailers in retailer group $k,\left\{1,2, \ldots, N_{k}\right\}$. Now, let $\mathbf{S}_{N_{k}}$ denote the vector of all order-up-to levels within retailer group $k$, $\left\{S_{1}, \ldots, S_{N_{k}}\right\}$, and $\mathbf{B}_{N_{k}}\left(t_{0}\right)$ denote the vector of backordered units to each retailer in retailer group $k$ at $t_{0},\left\{B_{1}\left(t_{0}\right), \ldots, B_{N_{k}}\left(t_{0}\right)\right\}$. The total costs for retailer group $k, T C_{k}\left(R_{0}, \mathbf{S}_{N_{k}}, T_{k}\right)$, for any values of $R_{0}, S_{N_{k}}$ and $T_{k}$ can then be seen as a sum over weighted averages of costs for all possible backorder combinations:

$$
T C_{k}\left(R_{0}, \mathbf{S}_{N_{k}}, T_{k}\right)=\sum_{b_{1}=0}^{\infty} \ldots \sum_{b_{k}=0}^{\infty} P\left\{\mathbf{B}_{N_{k}}\left(t_{0}\right)=\left\{b_{1}, \ldots, b_{k}\right\}\right\} T C_{k}\left(\mathbf{S}_{N_{k}}, T_{k} \mid \mathbf{B}_{N_{k}}\left(t_{0}\right)=\left\{b_{1}, \ldots, b_{k}\right\}\right) .
$$

This gives us for any value of $R_{0}$

$$
\begin{aligned}
T & C_{k}\left(R_{0}, \mathbf{S}, T_{k}\right) \geq \min _{T_{k}, \mathbf{S}_{N_{k}}}\left[T C_{k}\left(R_{0}, \mathbf{S}, T_{k}\right)\right] \\
= & \min _{T_{k}, \mathbf{S}_{N_{k}}}\left[\sum_{b_{1}=0}^{\infty} \ldots \sum_{b_{k}=0}^{\infty} P\left\{\mathbf{B}_{N_{k}}\left(t_{0}\right)=\left\{b_{1}, \ldots, b_{k}\right\}\right\} T C_{k}\left(\mathbf{S}_{N_{k}}, T_{k} \mid \mathbf{B}_{N_{k}}\left(t_{0}\right)=\left\{b_{1}, \ldots, b_{k}\right\}\right)\right] \\
\geq & \sum_{b_{1}=0}^{\infty} \ldots \sum_{b_{k}=0}^{\infty} P\left\{\mathbf{B}_{N_{k}}\left(t_{0}\right)=\left\{b_{1}, \ldots, b_{k}\right\}\right\} \min _{T_{k}, \mathbf{S}_{N_{k}}}\left[T C_{k}\left(\mathbf{S}_{N_{k}}, T_{k} \mid \mathbf{B}_{N_{k}}\left(t_{0}\right)=\left\{b_{1}, \ldots, b_{k}\right\}\right)\right] \\
= & \sum_{b_{1}=0}^{\infty} \ldots \sum_{b_{k}=0}^{\infty} P\left\{\mathbf{B}_{N_{k}}\left(t_{0}\right)=\left\{b_{1}, \ldots, b_{k}\right\}\right\} \min _{T_{k}, \mathbf{S}_{N_{k}}}\left[T C_{k}\left(\mathbf{S}_{N_{k}}, T_{k} \mid \mathbf{B}_{N_{k}}\left(t_{0}\right)=\{0, \ldots, 0\}\right)\right] \\
= & \min _{T_{k}, S_{i} \forall i \in N_{k}}\left(T C_{k} \mid B_{i}\left(t_{0}\right)=0, \forall i \in N_{k}\right) .
\end{aligned}
$$

The first equality follows from (B18). The second inequality is a consequence of relaxing the constraint forcing the same values of $T_{k}$ and $\mathbf{S}_{N_{k}}$ for all values of $\mathbf{B}_{N_{k}}\left(t_{0}\right)$. The second equality follows from (B17) and the fact that only the retailer costs are affected by the backorder distribution. The last equality follows directly as probabilities must sum to 1, completing the proof of (34) 


\section{Appendix C: A Small Numerical Example}

To illustrate the analysis we consider a system consisting of 3 retailers belonging to 2 retailer groups; retailers $\{1,2\}$ constitute the first group (and therefore have equal shipment intervals) and retailer 3 the second. Each retailer face compound Poisson demand with a logarithmic compounding distribution, i.e. with a variance to mean ratio of the demand at retailer $i$ of $\rho_{i}=\operatorname{Var}\left[D_{i}\right] / E\left[D_{i}\right]$ we have: $P\left\{Y_{i}=y\right\}=-\alpha_{i}^{y} /\left(\ln \left(1-\alpha_{i}\right) y\right)$ and $\lambda_{i}=-E\left[D_{i}\right]\left(1-\alpha_{i}\right) \ln \left(1-\alpha_{i}\right) / \alpha_{i}$, where $\alpha_{i}=1-\rho_{i}^{-1}$. The considered problem parameters are presented in Table 2.

Table 2: Values for parameters and decision variables in the illustrative example

\begin{tabular}{ccccc}
\hline \hline$E\left[D_{i}\right]=\{1,1,1\}$ & $R_{0}=-2$ & $T_{i}=\{0.5,0.5,1\}$ & $L_{i}=\{0.5,0.5,1,0.5\}$ & $\beta_{i}=\{10,10,10\}$ \\
$\rho_{i}=\{4,2,1.5\}$ & $Q_{0}=5$ & $S_{i}=\{4,4,4\}$ & $h_{i}=\{1,1,1,1\}$ & $\omega_{k}=\{2,2\}$ \\
\hline
\end{tabular}

As seen in Section 3.2, to analyze retailers $i(i=1,2,3)$ we need to determine the probability of $r$ warehouse backorders designated to retailer $i, P\left\{B_{i}\left(t_{0}\right)=r\right\}$, for all $r=\left[0, S_{i}-1\right]$, and the expected amount of backorders to retailer $i, E\left[B_{i}\left(t_{0}\right)\right]$. The computations are based on the analysis in Section 3.3. Two examples of the backorder distribution to retailer 1 conditioned on the inventory positions are $\left\{P\left\{B_{1}\left(t_{0}\right)=r \mid I P_{0}\left(t_{0}-L_{0}\right)=-1\right\}, r=0,1,2,3\right\}=\{0.607,0.262,0.057,0.028\}$ and $\left\{P\left\{B_{1}\left(t_{0}\right)=r \mid I P_{0}\left(t_{0}-L_{0}\right)=3\right\}, r=0,1,2,3\right\}=\{0.942,0.023,0.012,0.008\}$. Taking the average over all possible inventory positions $\left(I P_{0}\left(t_{0}-L_{0}\right)=[-1,3]\right)$ we get the steady state distributions of the backorders designated to each retailer, presented in Table 3. Exemplifying the Remark on page 20, Table 3 also presents the expected backorders designated to each retailer and illustrates their disproportions to the demand per time unit. One result of this is that the delay in the expected replenishment lead time to retailer 1 , caused by backorders at the central warehouse, will be higher than the delay experienced by retailers 2 and 3 .

Table 3: Distribution of warehouse backorders designated to each retailer

\begin{tabular}{c|cc}
\hline \hline Retailer $(i)$ & $\left\{P\left\{B_{i}\left(t_{0}\right)=r\right\}, r=0,1,2,3\right\}$ & $E\left[B_{i}\left(t_{0}\right)\right]$ \\
\hline 1 & $\{0.824,0.096,0.032,0.017\}$ & 0.399 \\
2 & $\{0.773,0.144,0.048,0.020\}$ & 0.373 \\
3 & $\{0.754,0.165,0.054,0.018\}$ & 0.367 \\
\hline
\end{tabular}

Knowing the backorder distributions, the expected stock on hand, $E\left[I L_{i}^{+}\right]$, the expected backorders, $E\left[I L_{i}^{-}\right]$, and the fill rates, $\gamma_{i}$, at the retailers can be determined from (7), (9) and (10) respectively. Calculating the expected stock on hand at the central warehouse using (3) and (4), the total cost of the system can be determined from (2). The results, determined analytically by the suggested approach, and simulated in a discrete event simulation program (Extend), are presented in Table 4.

Table 4: Results from exact analysis and simulation (Sim)

\begin{tabular}{c|ccccc}
\hline \hline & $E\left[I L_{0}^{+}\right]$ & $E\left[I L_{i}^{+}\right]$ & $E\left[I L_{i}^{-}\right]$ & $T C$ & $\gamma_{i}(\%)$ \\
\hline Exact & 1.639 & $\{3.087,2.541,2.704\}$ & $\{0.236,0.165,0.071\}$ & 20.691 & $\{72.6,79.5,88.1\}$ \\
$\operatorname{Sim}^{1}$ & 1.639 & $\{3.087,2.541,2.704\}$ & $\{0.236,0.165,0.071\}$ & 20.691 & $\{72.6,79.5,88.1\}$ \\
\hline \multicolumn{5}{c}{${ }^{1}$ The Standard deviations of the simulated results were $<0.001}$.
\end{tabular}




\section{Appendix D: Numerical Study}

For all 128 problem settings defined in Section 5, the shipment intervals, $T_{k} \forall k \in K$, the reorder points at the central warehouse, $R_{0}$, and the order up to levels at the retailers, $S_{i} \forall i \in N$, are optimized using the method described in Section 4. For optimizing the shipment intervals we have used a step size of of 0.01 time units. The complete results for all settings are available from the authors upon request. Table 5 summarizes the results in terms of average effects on: the optimal total cost, $T C^{*}$, the optimal reorder point at the central warehouse, $R_{0}^{*}$, the average of the optimal order-up-to level at the retailers, $S_{i}^{*}$, and the average of the optimal shipment intervals, $T_{k}^{*}$. It also includes the relative difference between the heuristic shipment intervals and the optimal, $\Delta T$, the relative increase in the associated costs, $\Delta C$, the difference between the upper bound on $R_{0}, R_{0}^{u}$, and the optimal value, $R_{0}^{*}$, $\Delta R_{0}^{u}$, the difference between the optimal value on $T_{k}, T_{k}^{*}$, and the lower bound, $T_{k}^{l}, \Delta T_{k}^{l}$, and the difference between the upper bound on $T_{k}, T_{k}^{u}$, and the optimal value, $T_{k}^{*}, \Delta T_{k}$. The results associated with $\rho_{i}=1$ are averages across all 64 problems where $\rho_{i}=1$, and analogously for all other parameters.

Table 5: Average results for the test series, for low and high values of $N, \rho_{i}, \beta_{i}, \omega_{k}, L_{0}, L_{i}$ and $Q_{0}$ as well as averages over all problems and minimum and maximum values

\begin{tabular}{c|ccccccccc}
\hline \hline & $E\left[T C^{*}\right]$ & $E\left[R_{0}^{*}\right]$ & $E\left[S_{i}^{*}\right]$ & $E\left[T_{k}^{*}\right]$ & $E[\Delta C]$ & $E[\Delta T]$ & $E\left[\Delta R_{0}^{u}\right]$ & $E\left[\Delta T_{k}^{l}\right]$ & $E\left[\Delta T_{k}^{u}\right]$ \\
\hline \hline$\rho_{i}{ }^{1}=1$ & 82.02 & 19.11 & 15.08 & 3.24 & $0.12 \%$ & $-0.60 \%$ & 4.91 & 2.05 & 15.81 \\
$\rho_{i}{ }^{1}=5$ & 141.00 & 20.67 & 23.75 & 3.05 & $0.16 \%$ & $6.57 \%$ & 9.92 & 2.33 & 31.28 \\
$\beta_{i}=10$ & 93.66 & 18.19 & 15.88 & 3.30 & $0.08 \%$ & $-2.27 \%$ & 7.42 & 2.21 & 18.88 \\
$\beta_{i}=100$ & 129.37 & 21.59 & 22.94 & 2.99 & $0.21 \%$ & $8.24 \%$ & 7.41 & 2.17 & 28.21 \\
$\omega_{k}=10$ & 81.96 & 21.25 & 15.97 & 1.54 & $0.13 \%$ & $1.90 \%$ & 7.28 & 1.25 & 19.07 \\
$\omega_{k}=100$ & 141.07 & 18.53 & 22.85 & 4.75 & $0.15 \%$ & $4.07 \%$ & 7.55 & 3.13 & 28.02 \\
$N=3$ & 84.78 & 10.86 & 20.45 & 3.68 & $0.15 \%$ & $3.18 \%$ & 6.53 & 2.52 & 25.82 \\
$N=6$ & 138.24 & 28.92 & 18.38 & 2.61 & $0.14 \%$ & $2.79 \%$ & 8.30 & 1.86 & 21.27 \\
$L_{0}=1$ & 108.36 & 1.27 & 18.86 & 3.11 & $0.17 \%$ & $4.41 \%$ & 5.06 & 2.11 & 22.05 \\
$L_{0}=5$ & 114.66 & 38.52 & 19.96 & 3.18 & $0.11 \%$ & $1.56 \%$ & 9.77 & 2.27 & 25.04 \\
$L_{i}=1$ and 2 & 107.98 & 20.45 & 17.26 & 3.10 & $0.18 \%$ & $4.94 \%$ & 7.48 & 2.13 & 22.96 \\
$L_{i}=2$ and 4 & 115.04 & 19.33 & 21.57 & 3.19 & $0.11 \%$ & $1.03 \%$ & 7.34 & 2.26 & 24.14 \\
$Q_{0}=2$ & 110.40 & 24.50 & 19.13 & 3.13 & $0.15 \%$ & $3.49 \%$ & 6.33 & 2.15 & 22.96 \\
$Q_{0}=20$ & 112.63 & 15.28 & 19.69 & 3.16 & $0.14 \%$ & $2.48 \%$ & 8.50 & 2.23 & 24.13 \\
\hline \hline Average & 111.51 & 19.89 & 19.41 & 3.15 & $0.14 \%$ & $2.99 \%$ & 7.41 & 2.19 & 23.55 \\
\hline Minimum & 31.61 & -10 & 7.67 & 1.09 & $0.00 \%$ & $-9.79 \%$ & 0 & 0.91 & 7.05 \\
Maximum & 264.19 & 68 & 38.33 & 6.11 & $0.66 \%$ & $18.60 \%$ & 24 & 4.23 & 51.26 \\
\hline
\end{tabular}

${ }^{1} \rho_{i}=1$ corresponds to Poisson demand at all retailers and $\rho_{i}=5$ to compound Poisson demand with logarithmic compounding distributions so that the variance-to-mean ratio of the demand per time unit at retailer $i$ is $5 \forall i \in N$.

Focusing first on the computational aspects and the optimality bounds, it is relevant to know that the optimization times for studied problems were between 0.2 and 120 minutes on a Dell Latitude 6400 lap top. The parameters that seem to affect the computational times the most are $\lambda_{0}, L_{0}, N$ and $K$. An important observation is that the fairly time consuming calculations of the backorder distributions at the central warehouse only needs to be computed once for each value of $R_{0}$ (in a given problem). Especially for the computationally more demanding problems, most of the computational time was spent on calculating these distributions. An explanation for this is that the complexity of determining these distributions increase with $R_{0}+Q_{0}$. As a result, the upper bound on the reorder level at the central warehouse, $R_{0}^{u}$ is the most important bound. Table 5 shows that this bound is rather tight, exceeding the optimal value $R_{0}^{*}$ by on average with only 7.41 units. It is especially tight in the cases where $\rho_{i}=1$ (on average only 4.91 units above optimum) and when $L_{0}=1$ (on average only 5.06 units above optimum). In fact, in the 32 problem settings investigated, where $\rho_{i}=1$ and $L_{0}=1$, 
$E\left[\Delta R_{0}^{u}\right]=3.53$. The fact that $E\left[\Delta R_{0}^{u}\right]$ is lower in systems where $L_{0}$ is lower is intuitive, as the optimal reorder level, $R_{0}^{*}$, is much lower for these systems. The effect of $\rho_{i}$ on the performance of the bound is perhaps less obvious. In order to explain this, recall that this bound is based on an estimation of the minimum costs at the retailers, $T C_{k}^{l}$, that assumes no backorders at the central warehouse (see Proposition 4). When the demand has a higher variance-to-mean ratio, a higher $R_{0}$-value is required for reaching this situation and the bound will therefore become looser.

Turning to the bounds on $T_{k}$, Table 5 shows that the lower bound is on average 2.19 time units below the optimal value (which is on average 3.15). The upper bound is looser as it is on average 23.55 time units above the optimal value. These bounds play a less important role in reducing the computational time than the upper bound on $R_{0}^{*}$.

Shifting our attention to the EOI heuristic, Marklund (2011) shows that it performs very well for Poisson demand. Based on Table 5 this seems to hold also for compound Poisson demand. The expected relative cost increase for all problem settings is only $0.14 \%$, although the heuristic tends to overestimate the optimal shipment intervals with on average $2.99 \%$. The relative cost increase is also only slightly higher in the systems where the variability in the demand is high $0.16 \%$ compared to $0.12 \%$ for the Poisson systems. There is a stronger tendency to overestimate the shipment intervals for the systems where $\rho_{i}=5(E[\Delta T]=6.57 \%)$, but because the total costs are much higher in these systems, the relative increase is still small. The parameter that seems to have the biggest influence on the performance of the heuristic is, in fact, the backorder costs. In systems where the backorder costs are high $E[\Delta C]=0.21 \%$. An explanation may be the desire to increase the flexibility in these systems by reducing the shipment intervals (the shipment intervals are overestimated by $8.24 \%$ in systems where $\beta_{i}=100$ when using the heuristic).

Considering the behavior of the optimal solutions, Table 5 illustrates that when the variability increases (i.e. comparing $\rho_{i}=1$ to $\rho_{i}=5$ ), the biggest difference in the control parameters is seen in the orderup-to levels of the retailers, which increase from on average 15.08 to 23.75. However there are also effects on the shipment intervals and reorder points at the central warehouse. The optimal warehouse reorder point increases from on average 19.11 to 20.67, thus raising the amount of available units at the central warehouse to handle the variability. The shipment intervals decrease from on average 3.24 to 3.05 with the effect that there is more flexibility in the system (the system can react faster if there is a big order at a specific retailer). Similar effects can be seen when increasing the backorder cost $\left(\beta_{i}\right)$. The largest effect on the the optimal control parameters is an increase in the average order-up-to levels at the retailers from 15.88 to 22.94 . However, we also see an increase in the average reorder point at the central warehouse from 18.19 to 21.59 and a decrease in the average shipment intervals (from 3.30 to 2.99 ), increasing the flexibility.

With regards to the shipment costs, we can see from Table 5 that as $\omega_{k} \forall k$ increase, the system reacts by increasing the shipment intervals (from on average 1.54 to 4.75). Moreover, the order-up-to levels at the retailers need to be raised accordingly in order to ensure stock for a longer replenishment cycle. Maybe less intuitively, the average optimal reorder points at the central warehouse decreases for these systems. This can be explained by the fact that increased shipment intervals results in longer replenishment lead times to the retailers, which may reduce the relative impact of inventory pooling at the central warehouse. Another contributing factor may be that the consolidation stock at the central warehouse increases with the shipment intervals. Thus, in order to avoid too much stock at the warehouse, the reorder point is reduced. For the other parameters, $N, L_{0}, L_{i}$ and $Q_{0}$, the behavior of the optimal solutions did not offer any insights beyond the obvious. 